\title{
Local Community Detection in Multilayer Networks
}

\author{
Roberto Interdonato • Andrea Tagarelli • \\ Dino Ienco • Arnaud Sallaberry - Pascal \\ Poncelet
}

\begin{abstract}
The problem of local community detection in graphs refers to the identification of a community that is specific to a query node and relies on limited information about the network structure. Existing approaches for this problem are defined to work in dynamic network scenarios, however they are not designed to deal with complex real-world networks, in which multiple types of connectivity might be considered. In this work, we fill this gap in the literature by introducing the first framework for local community detection in multilayer networks (ML-LCD). We formalize the ML-LCD optimization problem and provide three definitions of the associated objective function, which correspond to different ways to incorporate within-layer and across-layer topological features. We also exploit our framework to generate multilayer global community structures. We conduct an extensive experimentation using seven real-world multilayer networks, which also includes comparison with state-ofthe-art methods for single-layer local community detection and for multilayer global community detection. Results show the significance of our proposed methods in discovering local communities over multiple layers, and also highlight their ability in producing global community structures that are better in modularity than those produced by native global community detection approaches.
\end{abstract}

R. Interdonato

University of Calabria, Rende (CS), Italy, E-mail: rinterdonato@dimes.unical.it

A. Tagarelli

University of Calabria, Rende (CS), Italy, E-mail: andrea.tagarelli@unical.it

D. Ienco

IRSTEA, UMR TETIS, Montpellier, France, E-mail: dino.ienco@irstea.fr

A. Sallaberry

LIRMM - Université Paul Valéry, Montpellier, France, E-mail: arnaud.sallaberry@lirmm.fr P. Poncelet

LIRMM - Université de Montpellier, Montpellier, France, E-mail: pascal.poncelet@lirmm.fr 


\section{Introduction}

Community detection is a classic problem in network science and related fields, which has been traditionally addressed with the aim of determining an organization of a given network into subgraphs that express dense groups of nodes well-connected to each other (Newman and Girvan 2004). This corresponds to an optimization problem that is global as it requires knowledge on the whole network structure. The problem is known to be computationally difficult to solve, while its approximate solutions have to cope with both accuracy and efficiency issues that become more severe as the network increases in size. Large-scale, web-based environments have indeed traditionally represented a natural scenario for the development and testing of effective community detection approaches. Further challenges correspond to the emergence of processing complex real-world network systems, which are indeed pervasive in many fields of science (Mucha et al. 2010, Carchiolo et al. 2010; Tang et al. 2012, Kivela et al.|2014, Loe and Jensen 2015:|Kim and Lee 2015). In this regard, multilayer network models provide a powerful and more realistic tool for the analysis of such complex systems, enabling an in-depth understanding of the characteristics and dynamics of multiple, interconnected types of node relations and interactions (Cai et al. 2005, Berlingerio et al. 2013, Dickison et al. 2016).

However, one important aspect to consider is that we might often want to identify the personalized network of social contacts of interest to a single user only: to this aim, we would seek to determine the expanded neighborhood of that user which forms a densely connected, relatively small subgraph. This is known as local, or node-centric, community detection problem (Clauset 2005: Chen et al. 2009), whose general objective is, given limited information about the network, to identify a community structure which is centered on one or few seed users. The development of methods that can identify querydependent local communities is beneficial for any scenario in which computing a global community structure is not feasible (e.g., because the whole network information is not available at processing time), or it is not required (e.g., the communities are to be computed only for a subset of target users). As an intuitive practical example, if we want to simply check whether two users in a network belong to the same community, a global community detection method would process the whole network, while a local approach can efficiently discover the two communities of interest by accessing and manipulating only a relatively small portion of the network. This reduction of memory-footprint requirements by local community detection methods also enables an efficient processing of dynamic networks, whose structure may change over time (e.g., the insertion of new nodes and edges can be handled at each step of exploration); yet, local approaches can be useful to cope with privacy and access restriction issues that typically arise from policies adopted in most online social networks (e.g., limitation in the number of queries per day, permission to extract only the ego network of a limited number of seed nodes, and so on).

In the last few years, we have been witnessing an increasing interest towards the local community detection problem (Chen et al. 2009; Branting 
2012 Fagnan et al. 2014, Zakrzewska and Bader 2015; Li et al. 2015). Surprisingly, the problem has been mainly investigated by focusing on networks that are built on a single node-relation type or context. However, this is not the case in many situations. For instance, in social computing, an individual often has multiple accounts across different social networks, and in fact it has nowadays become important to link distributed user profiles belonging to the same user from multiple platforms (Kim and Lee 2015; Loe and Jensen 2015). An alternative scenario is obtained by considering that relations of different types can be available for the same population of a social network (Dickison et al. 2016); these might include online as well as offline (i.e., real-life) relations, such as followship, like/comment interactions, working relationship, lunch relationship, etc. Both scenarios can effectively be represented using a multilayer network model. Dealing with multiple graph-relation dimensions for a set of entities makes the previously discussed emergence of using a local community detection approach, as well as the reduction of memory requirements, even more evident and important. In general, several questions may arise, such as:

- How can we profitably use the various relations in which an individual is involved to discover her/his own community?

- How do the different relations affect the size and form of a multilayer local community being discovered?

- What advantages does the development of a method for multilayer local community detection may bring with respect to single-layer local community detection as well as to multilayer global community detection?

Contributions. In this work we aim to answer the above questions, by contributing a framework for the novel problem of local community detection in a multilayer network. To the best of our knowledge, we are the first to bring the local community detection problem into the context of multilayer networks since all previous works address the multilayer community detection task from a global point of view (e.g., (Mucha et al. 2010; Tang et al. 2012; Papalexakis et al. 2013 Kuncheva and Montana 2015; Kim and Lee 2015; Loe and Jensen 2015)). More in detail, we summarize our contributions as follows.

- We introduce and formalize the problem of local community detection for multilayer networks (ML-LCD), following an unsupervised paradigm.

- We provide three definitions of the objective function in the ML-LCD problem, which correspond to different ways to incorporate within-layer and across-layer topological features.

- To enable comparative evaluation with global community detection methods in multilayer networks, we exploit our ML-LCD framework to generate multilayer global community structures.

- We present an extensive experimentation of ML-LCD, using seven realworld multilayer networks. We assess the meaningfulness of our methods and analyze their different behaviors in identifying multilayer local communities. Moreover, we have conducted two stages of comparative evaluation with state-of-the-art methods for (single-layer) local community detection 
as well as for global community detection in multilayer networks; remarkably, in the latter case, ML-LCD has shown to produce communities with comparable or even better multilayer modularity.

Remarkably, the proposed ML-LCD methods are designed to provide community solutions that can meet several criteria of significance and quality. More specifically, under the classification framework discussed in the survey work on multilayer community detection methods by Kim and Lee (2015), our methods meet all (but one, i.e., algorithm insensitivity) of the desired properties for multilayer community detection methods, namely: multiple layer applicability; consideration of each layer's importance (this is in particular embedded in the first of our methods, but it can in principle be brought to the other methods as well); flexible layer participation (every local community has in general a different coverage of the layers' structure); no-layer-locality assumption (our local communities do not depend on initializations steps biased by a particular layer); independence from the order of layers; and overlapping layers (two or more local communities can share substructures over different layers).

The rest of the paper is organized as follows. Section 2 overviews related work, Section 3 describes our proposed local community detection methods for multilayer networks, Sections 4 and 5 present experimental evaluation, Section 6 concludes the paper and provides pointers for future research.

\section{Related Work}

We organize a brief discussion on related work into two parts: the first is devoted to community detection in multilayer networks, the second concerns local community detection methods.

Community detection in multilayer networks. Identifying a community structure in multilayer networks is a research topic that has gained considerable attention in the last few years (Kim and Lee 2015, Loe and Jensen 2015: Kivela et al. 2014). Early studies have focused on adaptations of the notion of modularity (Newman and Girvan 2004) to multilayer networks (Mucha et al. 2010: Carchiolo et al. (2010). Alternatively, new evaluation criteria have been designed specifically for multilayer networks (e.g., redundancy (Berlingerio et al. 2011)).

Tang et al. (2009) utilize structural feature extraction and cross-dimension integration to find a concise representation of features from the various layers (dimensions). A basic $k$-means clustering method is applied on the computed embedding to produce a community structure over the multilayer graph. In a subsequent work of the same authors (Tang et al. 2012), a utility integration criterion is introduced for computing utility matrices of a community detection method for each layer separately. Then it optimizes an objective function for the aggregated multilayer utility matrix. Kuncheva and Montana (2015) propose a community detection method for multilayer networks based on a multilayer random walk model. It first runs a different random walk for each layer, then a dissimilarity measure between nodes is obtained leveraging the 
per-layer transition probabilities, finally a hierarchical clustering method is used to produce the communities. The approach proposed by Hmimida and Kanawati (2015) leverages the concept of leaders in a network (Yakoubi and Kanawati 2014), i.e., nodes having higher degree centrality than most of their direct neighbors. These nodes, which first need to be identified over the whole network, form their own communities. An iterative local preference-merging method is then performed to compute and update the membership-preference vector of each node in function of preferences of its neighbors, so that each node will be assigned to the community defined by the leader ranked first in its preference vector. Other works have resorted to representation models such as third-order tensors (Papalexakis et al. 2013), hypergraphs (Michoel and Nachtergaele 2012), or transactional representation to support frequent pattern mining (Berlingerio et al. 2013).

Note that, by definition, all the above approaches address the problem of community detection from the conventional, global perspective, i.e., they assume to access the entire network structure in order to produce a partitioning of the network graph into a set of communities.

Local community detection. One of the earliest contribution to local community detection is the Clauset's framework (Clauset 2005), which is designed to explore the graph through local expansion starting from a seed node. Chen et al. (2009) exploit the Clauset framework to determine the quality of a community by comparing its internal versus external connectivity. Branting (2012) compares different local community detection methods, which are organized into two broad categories, namely xenophobic and non-xenophobic approaches: the former try to maximize (resp. minimize) the internal (resp. external) connectivity, while the non-xenophobic algorithms discard the external connectivity. Fagnan et al. (2014) propose a local community strategy that accounts for the number of internal and external triads. While still exploiting the Clauset framework, it finally produces a non-overlapping global community structure. The approach presented by Zakrzewska and Bader (2015) employs a seed set expansion procedure that incrementally updates the community as the underlying graph changes. Kanawati (2015) evaluates the impact of applying different ways of combining multiple local community functions to identify the node-centric communities. The Lemon algorithm proposed by Li et al. (2015) exploits truncated random walks and approximate invariant subspace to discover a local community for any given seed set. The number of random walk steps is a key parameter, which should be set high enough to reach all the nodes in the target community, and at the same time low enough to not spread to an unnecessary bigger graph.

It should be emphasized that none of the above works is designed to deal with multilayer networks. Different mention concerns the work by Jeub et al. (2015), where the solution of a personalized PageRank is approximated for a local partition of the multilayer network in order to find communities. However, the approach assumes complete knowledge about the network structure, and the local perspective is intended as the way the random walk is personalized, which differs from identifying local communities using multilayer features. In 
this work, we aim to fill this gap in the literature by contributing the first framework for multilayer local community detection.

\section{Multilayer Local Community Detection}

In this section, we first describe the graph model used to represent a multilayer network. We formally state the Multilayer Local Community Detection (MLLCD) problem. Then, we formulate the multilayer local community functions and describe the algorithmic framework for identifying a local community centered around an input seed node. We finally discuss computational complexity aspects of the proposed ML-LCD methods.

\subsection{Multilayer network model}

We refer to the multilayer network model described in (Kivela et al.|2014). Let $\mathcal{L}=\left\{L_{1}, \ldots, L_{\ell}\right\}$ be a set of layers. Each layer corresponds to a given type of entity relation, or edge-label. Consider a set $\mathcal{V}$ of entities (e.g., users), then for each choice of entity in $\mathcal{V}$ and layer in $\mathcal{L}$, we need to indicate whether the entity is present in that layer. We denote with $V_{\mathcal{L}} \subseteq \mathcal{V} \times \mathcal{L}$ the set containing the entity-layer combinations in which an entity is present in the corresponding layer. The set $E_{\mathcal{L}} \subseteq V_{\mathcal{L}} \times V_{\mathcal{L}}$ contains the undirected links between such entitylayer pairs. We hence denote with $G_{\mathcal{L}}=\left(V_{\mathcal{L}}, E_{\mathcal{L}}, \mathcal{V}, \mathcal{L}\right)$ the multilayer network graph with set of nodes $\mathcal{V}$.

For every layer $L_{i} \in \mathcal{L}$, let $V_{L_{i}}=\left\{v \in \mathcal{V} \mid\left(v, L_{i}\right) \in V_{\mathcal{L}}\right\} \subseteq \mathcal{V}$ be the set of nodes in the graph of $L_{i}$, and $E_{L_{i}} \subseteq V_{L_{i}} \times V_{L_{i}}$ be the set of edges in $L_{i}$. To simplify notations, we will also refer to $V_{L_{i}}$ and $E_{L_{i}}$ as $V_{i}$ and $E_{i}$, respectively. Note that while entities (i.e., elements of $\mathcal{V}$ ) are not required to participate to all layers, however each entity has to appear in at least one layer, i.e., $\bigcup_{i \in 1 . . \ell} V_{L_{i}}=\mathcal{V}$. Moreover, the only inter-layer edges are regarded as "couplings" of nodes representing the same entity between different layers; in other terms, $E_{\mathcal{L}}$ can be seen as partitioned into the set of intra-layer edges and the set of inter-layer coupling edges, i.e., $E_{\mathcal{L}}=\left\{\left(\left(u, L_{i}\right),\left(v, L_{j}\right)\right) \mid u, v \in\right.$ $\left.\mathcal{V} \wedge L_{i}, L_{j} \in \mathcal{L} \wedge i=j\right\} \cup\left\{\left(\left(v, L_{i}\right),\left(v, L_{j}\right)\right) \mid v \in \mathcal{V} \wedge L_{i}, L_{j} \in \mathcal{L} \wedge i \neq j\right\}$.

\subsection{Problem statement}

Local community detection approaches generally implement some strategy that at each step considers a node from one of three sets, namely: the community under construction (initialized with the seed node), the shell of nodes that are neighbors of nodes in the community but do not belong to the community, and the unexplored portion of the network. Figure1 1 illustrates an example of multilayer local community identified over a multilayer network; in the figure, we also utilize terms boundary and core nodes to differentiate between the 


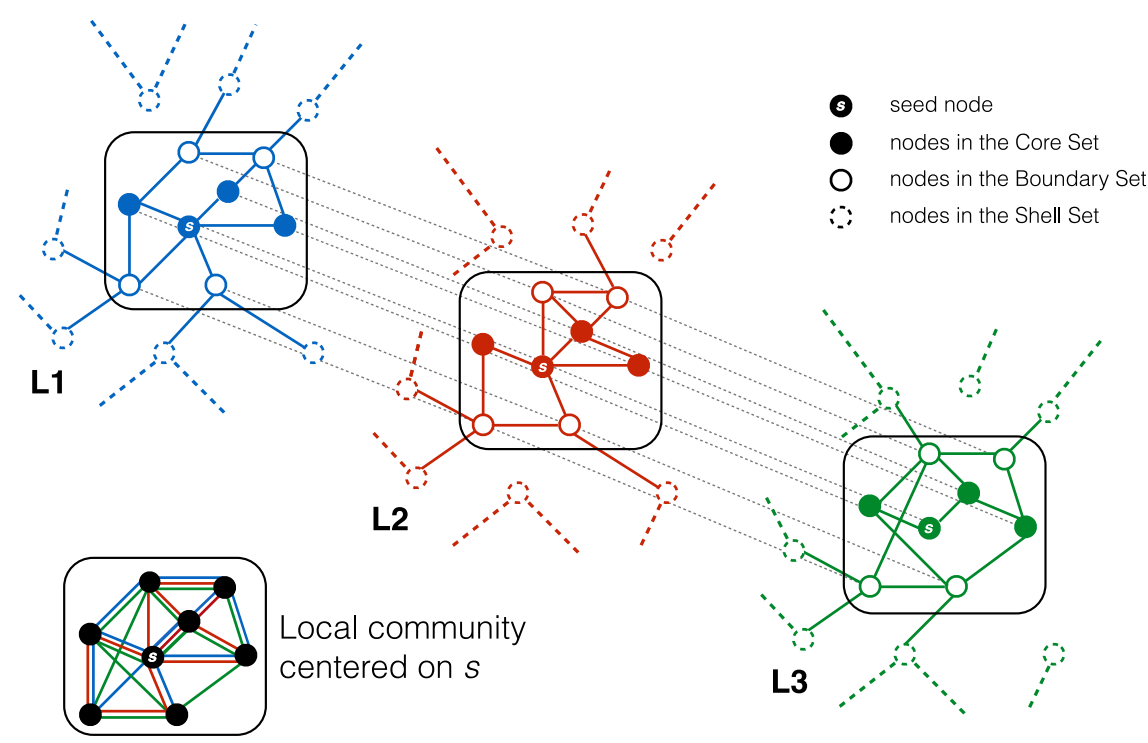

Fig. 1 A local community identified over an example 3-layer network. The local community (delimited by a rounded rectangle) is centered on a seed node $s$, while core nodes, boundary nodes, and shell nodes are denoted with filled, empty, and dotted circles, respectively. Dotted edges starting from the shell nodes point to unknown portions of the network. (Best viewed in color version, available in electronic format)

within-community nodes that have and do not have neighbors, respectively, in the shell set.

A key aspect in the task at hand is how to select the best node in the shell to add to the community to be identified. Most algorithms, which are designed to deal with simple (i.e., single-layer) network graphs, try to maximize a function in terms of the internal edges, i.e., edges that involve nodes in the community, and to minimize a function in terms of the external edges, i.e., edges to nodes outside the community. By accounting for both types of edges, nodes that are candidates to be added to the community being constructed are penalized in proportion to the amount of links to nodes external to the community Clauset 2005; Chen et al. 2009; Branting 2012; Fagnan et al. 2014).

In this work we follow the above general approach and extend it to identify local communities over a multilayer network as presented in the problem statement reported next.

Definition 1 (Multilayer Local Community Detection problem) Given a multilayer graph $G_{\mathcal{L}}=\left(V_{\mathcal{L}}, E_{\mathcal{L}}, \mathcal{V}, \mathcal{L}\right)$ with set of nodes $\mathcal{V}$, and a seed node $v_{0} \in \mathcal{V}$, find a subgraph $G_{\mathcal{L}}^{v_{0}} \subseteq G_{\mathcal{L}}$ that contains $v_{0}$ and maximizes the multilayer local community function $L C$ :

$$
G_{\mathcal{L}}^{v_{0}}=\underset{\substack{G=(V, E, \mathcal{V}, \mathcal{L}) \subseteq G_{\mathcal{L}} \\ \wedge v_{0} \in V}}{\operatorname{argmax}} L C(G)=\underset{\substack{(V, E, \mathcal{V}, \mathcal{L}) \subseteq G_{\mathcal{L}} \\ \wedge v_{0} \in V}}{\operatorname{argmax}} \frac{L C^{\text {int }}(G)}{L C^{\operatorname{ext}}(G)}
$$


where $L C^{i n t}(G)$ is a function proportional to the density of links among nodes within $G$, and $L C^{e x t}(G)$ is a function proportional to density of links between nodes within $G$ and nodes outside $G$.

It should be emphasized that our formulation accounts for the internal-toexternal connection density ratio rather than the absolute amount of internal and external links to the community. This is an important aspect since, as first analyzed in (Chen et al. 2009), it allows for alleviating the issue of inserting many weakly-linked nodes (i.e., outliers) into the local community being discovered.

In our setting, we also have to cope with the complexity of a multilayer network model. In this regard, in the following section we shall provide different definitions of our multilayer local community functions $L C^{i n t}(G)$ and $L C^{e x t}(G)$.

\subsection{Multilayer Local Community functions}

Given $G_{\mathcal{L}}=\left(V_{\mathcal{L}}, E_{\mathcal{L}}, \mathcal{V}, \mathcal{L}\right)$ and a seed node $v_{0}$, we denote with $C \subseteq \mathcal{V}$ the node set and with $E^{C} \subseteq E_{\mathcal{L}}$ the edge set of subgraph $G_{\mathcal{L}}^{v_{0}}$ corresponding to the local community built around node $v_{0}$; moreover, when the context is clear, we will use $C$ to refer to the local community subgraph. Symbol $E_{i}^{C}=\left\{(u, v) \mid \exists\left(\left(u, L_{i}\right),\left(v, L_{i}\right)\right) \in E^{C}\right\}$ will be used to specialize $E^{C}$ for edges in the community that correspond to a given layer $L_{i}$.

As discussed in the previous section, for a local community being constructed, the shell set refers to nodes external to the community that are neighbors of nodes in the community, and these within-community neighbors of shell nodes are also called boundary nodes. We define the shell set of $C$ as:

$$
S=\left\{v \in \mathcal{V} \backslash C \mid \exists\left(\left(u, L_{i}\right),\left(v, L_{j}\right)\right) \in E_{\mathcal{L}} \wedge u \in C\right\}
$$

and the boundary set of $C$ as:

$$
B=\left\{u \in C \mid \exists\left(\left(u, L_{i}\right),\left(v, L_{j}\right)\right) \in E_{\mathcal{L}} \wedge v \in S\right\} .
$$

Moreover, we denote with $E^{B}=\left\{(u, v) \mid\left(\left(u, L_{i}\right),\left(v, L_{j}\right)\right) \in E_{\mathcal{L}} \wedge u \in\right.$ $B \wedge v \in S\}$ the set of edges outgoing from $C$, and for any layer $L_{i}, E_{i}^{B}=$ $\left\{(u, v) \mid \exists\left(\left(u, L_{i}\right),\left(v, L_{i}\right)\right) \in E^{B}\right\}$ as the portion of $E^{B}$ corresponding to edges of layer $L_{i}$.

We devise different ways for completely specifying the internal-to-external connection density ratio expressed in the objective function of the problem in Def. 1. In particular, we will consider two intuitive criteria:

- Extension of the local community functions to handle multiple layers. In Sect. 3.3.1. we accomplish this by linearly combining the layer-specific contributions to the connection density ratio. 
- Integration of multilayer-aware, "homophilic" bias to control the community expansion. Homophily explains the tendency of individuals to associate and bond with similar others. Therefore, we might also want to make the evaluation of a candidate node sensitive to the affinity of the node with some of the community members. The rationale here is to bias the choice of a node in terms of its (direct or indirect ) homophily with the community, rather than only considering its contribution to the internal-to-external connection density ratio. In Sects. 3.3 .2 and 3.3 .3 we pursue this goal by modeling a within-layer similarity-based factor and a cross-layer similaritybased factor, respectively, into the objective function.

\subsubsection{Layer-weighting-based local community functions}

In our first specification of the problem in Def. 1. we incorporate multilayer features in the local community functions. One intuitive way to do this is to account for the relevance of each of the layers, which brings to the following definition of layer-weighting-based local community functions.

Definition 2 (Layer-weighted local community functions) Given $G_{\mathcal{L}}=$ $\left(V_{\mathcal{L}}, E_{\mathcal{L}}, \mathcal{V}, \mathcal{L}\right)$ and a local community $C$, the layer-weighting-based local community internal function is defined as:

$$
L C^{i n t}(C)=\frac{1}{|C|} \sum_{v \in C} \sum_{L_{i} \in \mathcal{L}} \omega_{i}\left|E_{i}^{C}(v)\right|
$$

The layer-weighting-based local community external function is defined as:

$$
L C^{e x t}(C)=\frac{1}{|B|} \sum_{v \in B} \sum_{L_{i} \in \mathcal{L}} \omega_{i}\left|E_{i}^{B}(v)\right|
$$

where $\omega_{i}$ (for every $L_{i} \in \mathcal{L}$ ) are non-negative real-valued coefficients, with $\sum_{L_{i} \in \mathcal{L}} \omega_{i}=1$, which define a weighting scheme over the layers.

The layer weighting scheme based on coefficients $\omega_{i}$ is set by default to a uniform distribution. Alternatively, it can be specified following either in an unsupervised or a supervised way. For instance, assuming to have some external knowledge on the relevance of the layers, the weights would be defined proportionally, thus following a supervised criterion. On the other hand, using any other distribution reflecting some known or computable graph property of the nodes/edges in each layer, would correspond to an unsupervised approach.

\subsubsection{Within-layer similarity-based local community functions}

While accounting for the presence of multiple layers, we believe it is also important to control the community expansion based on a notion of similarity in the node linkage, which might express a homophilic factor in the definition of multilayer local community functions. 
To this end, two major requirements are: (i) how to choose the analytical form of the similarity function, and (ii) how to deal with the different, layerspecific connections that any two nodes might have in the multilayer graph. We address the first point in an unsupervised fashion, by resorting to any similarity measure that can express the topological affinity of two nodes in the graph. Concerning the second point, one straightforward solution is to determine the similarity between any two nodes focusing on each layer at a time. The above points are formally captured by the following definition.

Definition 3 (Within-layer similarity-based local community functions) Given $G_{\mathcal{L}}=\left(V_{\mathcal{L}}, E_{\mathcal{L}}, \mathcal{V}, \mathcal{L}\right)$ and a local community $C$, the within-layer similarity-based community internal relation is defined as:

$$
L C^{i n t}(C)=\frac{1}{|C|} \sum_{v \in C} \sum_{L_{i} \in \mathcal{L}} \sum_{\substack{(u, v) \in E_{i}^{C} \\ \wedge u \in C}} \operatorname{sim}_{i}(u, v)
$$

The within-layer similarity-based community external relation is defined as:

$$
L C^{e x t}(C)=\frac{1}{|B|} \sum_{v \in B} \sum_{L_{i} \in \mathcal{L}} \sum_{\substack{(u, v) \in E_{i}^{B} \\ \wedge u \in S}} \operatorname{sim}_{i}(u, v)
$$

where $\operatorname{sim}_{i}(u, v)$ denotes the similarity between nodes $u, v$ contextually to layer $L_{i}$.

As previously mentioned, we adopt an unsupervised approach to the evaluation of the similarity between two nodes $u, v$, by measuring the topological affinity of $u$ and $v$. We choose to accomplish this by resorting to any topologybased similarity measure between two node sets, or more generally, between their induced subgraphs. In this work, we regard the generic form of $\operatorname{sim}_{i}(u, v)$ as $\mathrm{f}\left(N_{i}(u), N_{i}(v)\right)$, where $\mathrm{f}$ denotes a function proportional to the similarity of two node sets $N_{i}(u), N_{i}(v)$, with $N_{i}(x)=\left\{y \in \mathcal{V} \mid(y, x) \in E_{i}\right\}$ as the set of neighbors of a node $x$ in layer $L_{i}$. In Section 4 we will discuss similarity measures used in our experimental evaluation.

\subsubsection{Cross-layer similarity-based local community functions}

One evident simplification in the previous definition is that it expresses the homophily between two nodes by quantifying the strength of their connections considering the layers on which they lay as separately to each other. This might also be a limitation in that the within-layer similarity-based method is not able to capture indirect homophily, i.e., ties between nodes that are not directly connected through the network: nevertheless, two nodes can still have some topological affinity even without the presence of an explicit edge in the network (e.g., having lots of friends in common while being unknown to each other). Based on this simple intuition, we provide our definition of cross-layer similarity-based local community functions. 
Definition 4 (Cross-layer similarity-based local community functions) Given $G_{\mathcal{L}}=\left(V_{\mathcal{L}}, E_{\mathcal{L}}, \mathcal{V}, \mathcal{L}\right)$ and a local community $C$, the cross-layer similaritybased community internal function is defined as:

$$
L C^{i n t}(C)=\frac{1}{|C|} \sum_{u, v \in C} \sum_{\substack{L_{i}, L_{j} \in \mathcal{L} \\ \wedge u \in V_{i}, v \in V_{j}}} \operatorname{sim}_{i, j}(u, v)
$$

The cross-layer similarity-based community external relation is defined as:

$$
L C^{e x t}(C)=\frac{1}{|B|} \sum_{v \in B, u \in S} \sum_{\substack{L_{i}, L_{j} \in \mathcal{L} \\ \wedge u \in V_{i}, v \in V_{j}}} \operatorname{sim}_{i, j}(u, v)
$$

where $\operatorname{sim}_{i, j}(u, v)$ denotes the similarity between $u$ in layer $L_{i}$ and $v$ in layer $L_{j}$.

Analogously to the previous definition, $\operatorname{sim}_{i, j}(u, v)$ corresponds to a topologybased similarity measure between the sets of neighbors of $u$ and $v$, which however in this case are located in different layers.

\subsection{Multilayer Local Community Identification Algorithm}

Algorithm 1 reports the pseudo-code of the general scheme of our proposed MultiLayer Local Community Detection (ML-LCD) methods.

The scheme takes as input the multilayer graph $G_{\mathcal{L}}$ and a seed node $v_{0}$, and computes the local community $C$ associated to $v_{0}$. Recall that knowledge on the topology of the multilayer graph is only partial in our setting; precisely, at any give time step, only the direct neighbors of a node are known in advance. Note also that the general scheme is actually instantiated in three algorithms, according to Def. 2, Def. 3 , or Def. 4 We will use notation MLLCD-lw, ML-LCD-wlsim, and ML-LCD-clsim to refer to the ML-LCD algorithm equipped with layer-weighting-based, within-layer similarity-based, and cross-layer similarity-based local community functions, respectively.

At the beginning, the boundary set $(B)$ and the community $(C)$ are initialized with the starting seed, while the shell set $(S)$ is initialized with the neighborhood set of $v_{0}$ considering all the layers in $\mathcal{L}$. The initial value of $L C(C)$ is computed (lines 3-4) according to one of the three approaches for the definition of the local community functions. The algorithm then starts expanding the node set $C$ (lines $5-19$ ). First, it evaluates nodes $v$ belonging to the current shell set $S$, and selects the vertex $v^{*}$ that corresponds to the maximum value of objective function $L C$ if the node would be added to $C$ (line 6). The choice of candidate node $v^{*}$ impacts on both $S$ and $B$ (lines $7-8)$ : node $v^{*}$ is removed from $S$ (since it has been examined), whereas all nodes in $B$ that have no other neighbors in $S$ than $v^{*}$ are removed from $B$. Since adding node $v^{*}$ to $C$ might not increase the current value of the objective function, the algorithm checks if (i) $v^{*}$ actually increases the quality of 


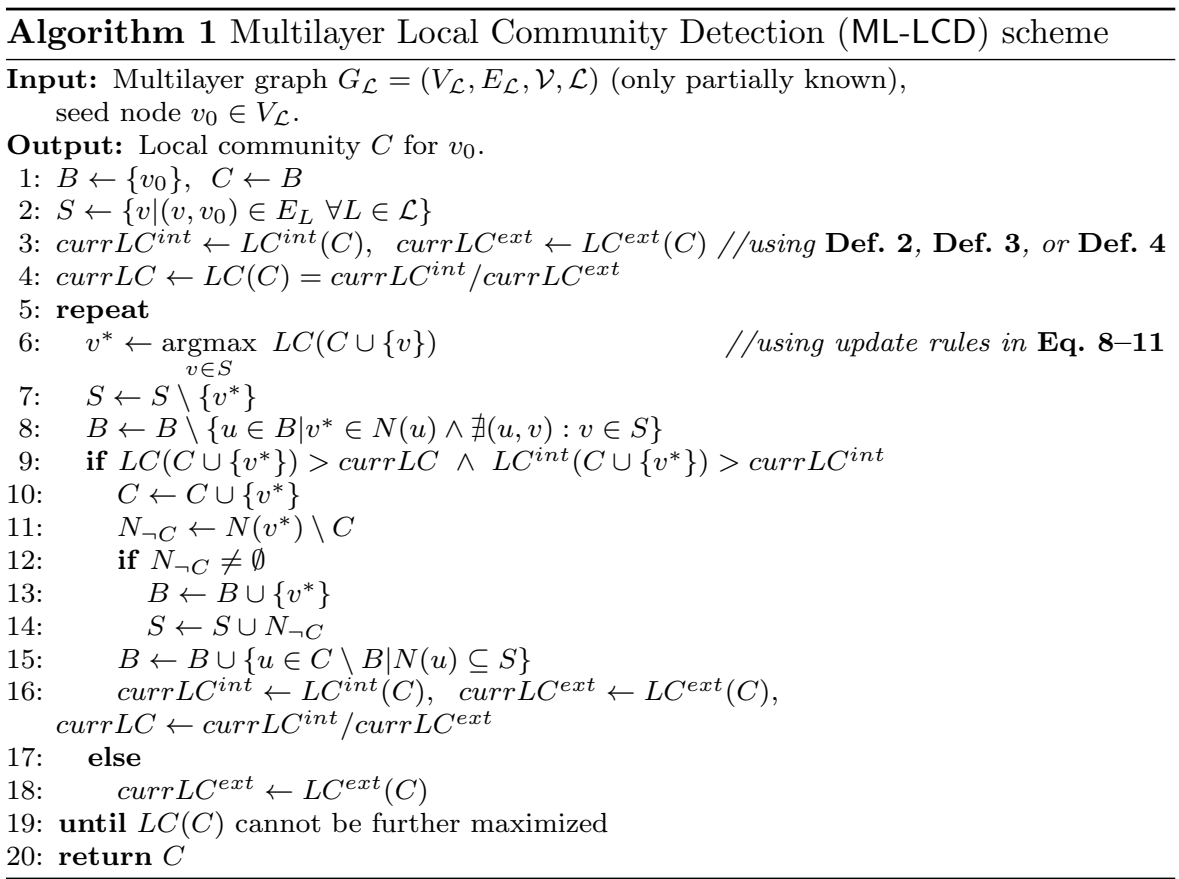

$C$ (i.e., $L C\left(C \cup\left\{v^{*}\right\}\right)>\operatorname{curr} L C$ ) and (ii) $v^{*}$ helps to strength the internal connectivity of the community (i.e., $\left.L C^{\text {int }}\left(C \cup\left\{v^{*}\right\}\right)>\operatorname{curr} L C^{\text {int }}\right)$. If both conditions are satisfied (line 9), node $v^{*}$ is added to $C$ and the following actions are taken: (i) $v^{*}$ is added to $B$ as long as it has neighbors that are not in $C$ (line 13), and the shell set is updated to contain these nodes (line 14); (ii) a further update to $B$ might be requested in case there are nodes in $C$ that now have neighbors in $S$ (line 15); (iii) $L C\left(C \cup\left\{v^{*}\right\}\right)$ becomes the new value of $\operatorname{curr} L C$. Otherwise, if node $v^{*}$ is not added to $C$, only $L C^{e x t}(C)$ is updated according to the changes in $S$ and $B$ (performed at lines 7-8). The algorithm terminates when no further improvement in $L C(C)$ is possible.

$L C^{\text {int }}$ and $L C^{\text {ext }}$ update formulas. Testing a candidate node $v \in S$ for possible insertion into $C$ (line 6) requires evaluation of $L C$ for an updated community $C \cup\{v\}$. To do this, we can avoid computing $L C^{i n t}$ and $L C^{e x t}$ from scratch by using the incremental update formulas reported next; in this regard, we use symbols $B_{v}$ and $S_{v}$, where $B_{v} \subseteq B$ contains neighbors of $v$ in $B$ that have no other neighbors in $S$, and $S_{v}=N(v) \backslash C$ contains neighbors of $v$ that are not in $C$.

- ML-LCD-lw and ML-LCD-wlsim:

$$
L C_{v}^{i n t}=|C| L C^{i n t}+\sum_{u \in C} \sum_{\substack{L_{i} \in \mathcal{L} \\ \wedge(u, v) \in E_{i}^{C}}} \Gamma
$$




$$
L C_{v}^{e x t}=|B| L C^{e x t}+\sum_{u \in S_{v}} \sum_{\substack{L_{i} \in \mathcal{L} \\ \wedge\left(\left(u, L_{i}\right),\left(v, L_{i}\right)\right) \in E_{\mathcal{L}}}} \Gamma-\sum_{u \in B_{v}} \sum_{\substack{L_{i} \in \mathcal{L} \\ \wedge(u, v) \in E_{i}^{B}}} \Gamma
$$

where $\Gamma$ is a reference such that $\Gamma \equiv \omega_{i}$ in the case of ML-LCD-lw and $\Gamma \equiv \operatorname{sim}_{i}(u, v)$ in the case of ML-LCD-wlsim.

- ML-LCD-clsim:

$$
\begin{aligned}
L C_{v}^{\text {int }} & =|C| L C^{i n t}+\sum_{u \in C} \sum_{\substack{L_{i}, L_{j} \in \mathcal{L} \\
\wedge\left(\left(u, L_{i}\right),\left(v, L_{j}\right)\right) \in E_{\mathcal{L}}}} \operatorname{sim}_{i, j}(u, v) \\
L C_{v}^{e x t} & =|B| L C^{e x t}+\sum_{u \in S_{v}} \sum_{s \in S} \sum_{\substack{L_{i}, L_{j} \in \mathcal{L} \\
\wedge((u, i),(s, j)) \in E_{\mathcal{L}} \\
\operatorname{sim}_{i, j}(u, s)}} \operatorname{sim}_{i, j}(u, s)+ \\
& -\sum_{u \in B_{v}} \sum_{s \in S} \sum_{\substack{L_{i}, L_{j} \in \mathcal{L} \\
\wedge((u, i),(s, j)) \in E_{\mathcal{L}}}}
\end{aligned}
$$

\subsection{Computational complexity aspects}

We discuss here computational complexity aspects of ML-LCD. We introduce symbol $\Phi$ to denote the computational cost of any of the proposed local community functions, and symbol $d$ to indicate the maximum degree of a node in the network. We recall that $\ell=|\mathcal{L}|$ denotes the number of layers and $|C|$ denotes the size of the community being discovered.

In Algorithm 1, at each iteration the community size increases by one node until it reaches a certain size $|C|$, whereas the shell set $S$ size may vary over the iterations. In this regard, we introduce the quantity $(k \times d)$ as an upper bound of the size of $S$ at the current iteration, where $k$ corresponds to the size of the current community (proportional to the number of the current iteration). Note that this upper bound corresponds to the worst case in which all nodes in the current community are assumed to belong to the boundary set $B$ and all of the neighbor sets are disjoint.

Since the algorithm terminates in a number of iterations which is proportional to the size of the generated community, we state the following upper bound of the overall computational complexity of Algorithm 1 . $\mathcal{O}(d \times \Phi \times$ $\left.\sum_{k=1}^{|C|} k\right)$, which can be rewritten as $\mathcal{O}\left(|C|^{2} \times d \times \Phi\right)$.

Considering ML-LCD-lw, the characteristic operation has a cost proportional to the neighbors of any node $v$ in $C$ and the number of layers $\ell$, since the weights $\omega$ are known at constant time. Therefore, $\Phi=\mathcal{O}(\ell \times d)$, which leads to the cost of $\mathcal{O}\left(|C|^{2} \times d^{2} \times \ell\right)$ for ML-LCD-lw.

While following the same strategy as ML-LCD-lw, method ML-LCD-wlsim also requires the computation of similarity between any node $v$ and its neighbors. This implies at least comparison between the set of (direct) neighbors of 
Table 1 Main characteristics of the multilayer network datasets

\begin{tabular}{|l|c|c|c|c|c|c|}
\hline Dataset & \# Nodes & \# Edges & \#Layers & Density & $A_{\text {deg }}$ & $A_{\text {layer }}$ \\
\hline \hline Airlines & 417 & 3588 & 37 & 0.056 & 17.21 & 4.88 \\
AUCS & 61 & 620 & 5 & 0.114 & 20.33 & 3.67 \\
Biogrid & 38936 & 342599 & 7 & $4.8 \mathrm{e}-4$ & 17.6 & 1.9 \\
DBLP & 83901 & 159302 & 50 & $8.9 \mathrm{e}-4$ & 3.8 & 1.35 \\
RealityMining & 88 & 355 & 3 & 0.047 & 8.07 & 2.42 \\
RemoteSensing & 642 & 4341 & 5 & 0.006 & 13.52 & 4.19 \\
TW-YT-FF & 6407 & 74862 & 3 & $2.35 \mathrm{e}-3$ & 23.37 & 1.86 \\
\hline
\end{tabular}

$v$ and the set of (direct) neighbors of $u$. If we assume that the neighborhood lists are always ordered (based on their identifiers), the cost of set comparison becomes $\mathcal{O}(d \log d)$ - checking if an element is present in an ordered list $\operatorname{costs} \mathcal{O}(\log d)$ and this basic operation is repeated $d$ times. In this case, $\Phi=$ $\mathcal{O}\left(\ell \times d^{2} \log d\right)$. Therefore, the cost for ML-LCD-wlsim is $\mathcal{O}\left(|C|^{2} \times d^{3} \log d \times \ell\right)$.

The topology-unawareness of ML-LCD-clsim leads to an increase in the cost $\Phi$. Specifically, the cost becomes proportional to $|C|^{2}$ multiplied by the sum of the costs of functions $L C^{i n t}$ and $L C^{e x t}$. The latter is expressed in terms of size of $S$, which, in turn, is expressed in terms of size of the current community; therefore, in the worst case the cost of $L C^{e x t}$ is predominant over $L C^{i n t}$, thus we focus on $L C^{e x t}$. At each iteration of Algorithm 1, we might compare each node in the boundary $B$ (in the worst case, the whole $C$ ), to each node in the shell set $S$. Therefore, for ML-LCD-clsim, the overall cost is given by: $\mathcal{O}\left(\sum_{k=1}^{|C|} k \times d \times k \times d \times \ell^{2} \times d \log d\right)$, where the first term $(k \times d)$ is related to the basic cost, while the second term $(k \times d)$ refers to the specific topology-unawareness characteristic of ML-LCD-clsim. Rearranging the terms in the previous formula results in $\mathcal{O}\left(|C|^{3} \times d^{3} \log d \times \ell^{2}\right)$.

\section{Experimental Evaluation}

In the following, Section 4.1 summarizes the evaluation datasets, Section 4.2 introduces competing methods, and Section 4.3 describes the experimental settings.

\subsection{Data}

We used seven real-world multilayer network datasets, namely Airlines Cardillp et al. 2013), AUCS (Dickison et al. 2016), BIOGRID (Bonchi et al.|2014), DBLP (Boden et al. 2012), Reality Mining (Kim and Lee 2015||Bourqui et al. 2016), RemoteSensing, and TW-YT-FF (Dickison et al. 2016). Airlines describes airline companies operating in Europe. Nodes and edges represent airport locations and routes, respectively, and each layer corresponds to a different airline company. AUCS models relationships between employees of a University department considering five different aspects: coworking, having 
lunch together, Facebook friendship, offline friendship, and coauthorship. $B i$ ogrid is a protein-protein interaction network, where layers correspond to seven different types of interactions between proteins. In $D B L P$, nodes correspond to authors and layers represent the top-50 Computer Science conferences. Two authors are connected on a layer if they co-authored at least two papers together in a particular conference. Reality Mining contains human interaction data collected by the MIT Media Lab, where layers represent different media employed to communicate: subjects calling each other, friendship claims, and text message exchanges. RemoteSensing is a network derived from a remote sensing satellite image obtained from the SWH website ${ }^{1}$ Nodes correspond to segments of the image and edges of a certain type exists between two segments if they are adjacent and have the same value for a certain radiometric attribute (layer). $T W-Y T-F F$ is a cross-platform network built by exploiting the feature of FriendFeed as social media aggregator to align registered users who were also members of Twitter and YouTube. Nodes correspond to users and edges to friendships over the three different platforms as layers.

Table 1 summarizes main characteristics of our evaluation datasets. Node relations in all datasets are treated as symmetric. We denote with $A_{\text {deg }}$ the average degree of a node considering multiple edges, and with $A_{\text {layer }}$ the average number of layers in which a node is present. Note that Airlines and DBLP are rich in number of layers (resp. 37 and 50), and that Airlines, RemoteSensing, $A U C S$ and RealityMining have nodes, on average, involved in more than two layers (resp. 4.88, 4.19, 3.67 and 2.42).

\subsection{Competing methods}

Our evaluation focus is on understanding how the proposed local community detection methods perform on multilayer networks. Nevertheless, given the unavailability of competing methods for local community detection in multilayer networks, we devised two stages of comparative evaluation: (1) comparison with single-layer, local community detection, and (2) comparison with multilayer, global community detection approaches.

For the first stage of comparative evaluation, we resorted to the methods LCD (Chen et al. 2009) and Lemon (Li et al. 2015). The LCD approach is chosen since its strategy of identification of a local community is close to ours, while Lemon is a more recent, state-of-the-art method for local community detection. Since the methods are designed to deal with single-layer graphs, we applied each of them on the aggregate graph derived from the input multilayer network $G_{\mathcal{L}}$, i.e., a graph with set of nodes $\mathcal{V}$ and such that an edge exists between any two nodes that are connected in at least one layer in $G_{\mathcal{L}}$. While LCD is completely parameter free, for Lemon we used the default setting ${ }^{2}$ also

\footnotetext{
1 https://www.theia-land.fr/en/products/spot-world-heritage

2 As specified in the publicly available implementation from the repository at https: //github.com/YixuanLi/LEMON
} 
disabling the ground-truth option and the sampling for graphs with less than $1 \mathrm{k}$ nodes.

For the second stage of comparative evaluation, we used Locally Adaptive Random Transitions (LART) (Kuncheva and Montana 2015), Principal Modularity Maximization (PMM) (Tang et al. 2009) and Generalized Louvain (GL) (Mucha et al. 2010). Note that all such methods produce non-overlapping global community structures, while the local communities produced by MLLCD for various seed nodes can of course overlap to each other. Note also that LART and GL deal with node-layer pairs, thus allowing different instances of the same node to belong to more communities; to make the two methods comparable with all the other approaches, we apply a majority voting mechanism, so that a node is assigned to the community containing the majority of its instances.

Inferring Multilayer Global Community Structure. In order to compare our ML-LCD methods with global community detection methods, we propose a heuristic, hereinafter referred to as LocToGlob, to infer a global non-overlapping community structure from local solutions produced by our methods.

LocToGlob takes as input the multilayer graph $G_{\mathcal{L}}$ and yields a set of nonoverlapping communities. The algorithm works by iteratively performing a covering phase, which first computes the local community for a selected seed node, then updates the global community structure and the portion of graph that has not been processed yet. For the selection of seed node at each iteration of ML-LCD, we employ the simple heuristic (Fagnan et al. 2014), which first samples at random a node $v$ then selects the node with maximum degree among $v$ and its neighbors. The covering phase terminates when all nodes have been processed. Since this phase may produce singleton communities, a post-processing phase is performed to re-assign each of the nodes belonging to a singleton community to the (non-singleton) community for which adding the singleton produces the highest increase in the local community function.

\subsection{Experimental settings}

Each of our ML-LCD methods was carried out over all nodes in a network, by selecting one node at a time as seed. ML-LCD-wlsim and ML-LCD-clsim require the definition of a node similarity measure for a given layer $\left(\operatorname{sim}_{i}(\cdot, \cdot)\right)$ or couple of layers $\left(\operatorname{sim}_{i, j}(\cdot, \cdot)\right)$, respectively (cf. Section 3.3). We considered three alternative measures, as listed below:

- Jaccard similarity, i.e., $\operatorname{sim}_{i, j}(u, v)=\frac{\left|N_{i}(u) \cap N_{j}(v)\right|}{\left|N_{i}(u) \cup N_{j}(v)\right|}$, where $N_{i}(u)=\{v \in$ $\left.\mathcal{V} \mid(v, u) \in E_{i}\right\}$ denotes the set of neighbors of a node $u$ in layer $L_{i}$ (with $i=j$ for ML-LCD-wlsim).

- Cosine similarity, whereby the proportionality of shared neighborhood is smoothed to favor unbalanced neighborhoods to be compared: $\operatorname{sim}_{i, j}(u, v)=$ $\frac{\left|N_{i}(u) \cap N_{j}(v)\right|}{\sqrt{\left|N_{i}(u)\right|\left|N_{j}(v)\right|}}$ (with $i=j$ for ML-LCD-wlsim). 
- Triad-based similarity, which we define as a Jaccard similarity calculated on the set of 3-cliques to which any two nodes $u, v$ belong to: $\operatorname{sim}_{i, j}(u, v)=$ $\frac{\left|N_{i}(u) \cap N_{j}(v)\right|}{\left|T_{i}(u) \cup T_{j}(v)\right|}$, where $T_{i}(u)$ indicates the set of 3-cliques (or triads) to which node $u$ belongs to on layer $i$ ( $i=j$ for ML-LCD-wlsim). Please note that, since we take into account undirected graphs, the number of triads in common between two adjacent nodes is equivalent to the number of neighbors in common.

We will use notations $j a c, c o s$, and triads to refer to the above instantiations of function sim. For the layer weighting scheme in ML-LCD-lw, we assume uniform weights.

As concerns the evaluation of global community detection, we averaged performance results of LocToGlob and PMM over a relatively large number of runs (50), in order to reduce the bias due to their non-deterministic behavior. The number of desired communities, which is a further input in PMM, was set equal to the number of communities extracted by each of our LocToGlob variants based on ML-LCD. Performance results by LocToGlob variants and competing ones are assessed by means of the multilayer (or multislice) modularity measure proposed in (Mucha et al. 2010) $!^{3}$

\section{Results}

We organize the presentation of experimental results in three subsections: Section 5.1 provides an in-depth analysis of the communities identified by our MLLCD methods, whereas the subsequent two subsections contain comparative evaluation of ML-LCD with local community detection methods on aggregate graphs (Section 5.2) and with global, multilayer community detection methods (Section 5.3).

\subsection{Evaluation of ML-LCD methods}

We assessed the behavior of the proposed ML-LCD methods in terms of: (1) size of extracted local communities, (2) structural characteristics of the communities, (3) similarity between communities, (4) distribution of layers involved in each of the local communities, (5) community distribution over number of edges, (6) impact of similarity measures on ML-LCD-wlsim and ML-LCD-clsim, (7) overlap of communities generated by each of the methods, (8) efficiency analysis. In the following we will present results concerning each of the above evaluation aspects.

\footnotetext{
3 Experiments were carried out on an Intel Core i7-3960X CPU @3.30GHz, 64GB RAM machine. All our algorithms are written in Python. Original codes of competing methods are in Python (LART) and Matlab (PMM and GL).
} 
Table 2 Mean and standard deviation of the size of the communities identified by ML-LCD methods and by LCD and Lemon. Bold and underlined values correspond to the maximum (mean) size obtained by a ML-LCD method and all selected methods, respectively.

\begin{tabular}{|l||c|c|c||c|c|}
\hline Dataset & ML-LCD- $w$ w & ML-LCD-wlsim & ML-LCD-clsim & LCD & Lemon \\
\hline \hline Airlines & $\mathbf{3 3 . 6 2} \pm 33.57$ & $11.48 \pm 15.04$ & $20.42 \pm 21.38$ & $9.69 \pm 11.29$ & $\underline{73.34} \pm 15.83$ \\
AUCS & $\mathbf{1 1 . 2 3} \pm 3.40$ & $7.90 \pm 2.74$ & $9.10 \pm 3.30$ & $11.48 \pm 4.28$ & $\underline{\underline{55.52} \pm 11.48}$ \\
Biogrid & $\mathbf{1 7 . 6 5} \pm 53.27$ & $4.87 \pm 9.14$ & $8.80 \pm 38.71$ & $6.45 \pm 11.43$ & $\underline{\underline{61.88}} \pm 23.99$ \\
DBLP & $\mathbf{4 . 5 2} \pm 2.74$ & $3.67 \pm 2.16$ & $3.72 \pm 2.90$ & $3.53 \pm 2.43$ & $\underline{\underline{36.45} \pm 24.71}$ \\
RealityMining & $\mathbf{8 . 0 5} \pm 7.08$ & $3.37 \pm 1.77$ & $7.02 \pm 5.28$ & $5.23 \pm 4.31$ & $\underline{\underline{49.55} \pm 21.36}$ \\
RemoteSensing & $\mathbf{7 . 4 6} \pm 3.30$ & $5.66 \pm 2.53$ & $5.47 \pm 2.82$ & $7.98 \pm 3.44$ & $\underline{\underline{37.83} \pm 18.88}$ \\
TW-YT-FF & $15.54 \pm 29.14$ & $17.53 \pm 34.01$ & $\mathbf{1 7 . 9 8} \pm 21.05$ & $9.88 \pm 17.71$ & $\underline{\underline{70.75}} \pm 21.00$ \\
\hline
\end{tabular}

Table 3 Per-layer average path length and clustering coefficient of the communities identified by ML-LCD methods. Bold values correspond to the minimum per-dataset (mean) average path length and to the maximum per-dataset (mean) clustering coefficient.

\begin{tabular}{|l||cc||cc|}
\hline \multirow{2}{*}{ Dataset } & average path length & \multicolumn{2}{c|}{ clustering coefficient } \\
\cline { 2 - 5 } & mean sd & max & mean sd & max \\
\hline \hline \multicolumn{5}{|c|}{ ML-LCD-lw } \\
\hline Airlines & $0.881 \pm 0.143$ & $2.259 \pm 0.365$ & $\mathbf{0 . 0 7 7} \pm 0.088$ & $0.488 \pm 0.374$ \\
AUCS & $1.479 \pm 0.201$ & $1.978 \pm 0.207$ & $\mathbf{0 . 5 6 0} \pm 0.180$ & $0.801 \pm 0.138$ \\
Biogrid & $0.656 \pm 0.430$ & $6.049 \pm 0.767$ & $\mathbf{0 . 0 9 5} \pm 0.102$ & $1.000 \pm 0.000$ \\
DBLP & $0.041 \pm 0.029$ & $2.306 \pm 0.284$ & $\mathbf{0 . 0 1 6} \pm 0.011$ & $1.000 \pm 0.000$ \\
RealityMining & $1.409 \pm 0.108$ & $2.545 \pm 0.621$ & $0.276 \pm 0.112$ & $0.889 \pm 0.192$ \\
RemoteSensing & $1.474 \pm 0.048$ & $2.786 \pm 0.145$ & $0.471 \pm 0.082$ & $1.000 \pm 0.000$ \\
TW-YT-FF & $1.368 \pm 1.107$ & $4.515 \pm 2.250$ & $0.128 \pm 0.118$ & $1.000 \pm 0.000$ \\
\hline \hline \multicolumn{5}{|c|}{ ML-LCD-wlsim } \\
\hline Airlines & $\mathbf{0 . 2 2 2} \pm 0.168$ & $1.872 \pm 0.397$ & $0.022 \pm 0.027$ & $0.508 \pm 0.398$ \\
AUCS & $\mathbf{1 . 1 8 2} \pm 0.237$ & $1.880 \pm 0.364$ & $0.533 \pm 0.238$ & $0.938 \pm 0.087$ \\
Biogrid & $\mathbf{0 . 3 1 4} \pm 0.247$ & $3.839 \pm 0.655$ & $0.079 \pm 0.091$ & $1.000 \pm 0.000$ \\
DBLP & $0.030 \pm 0.021$ & $1.875 \pm 0.202$ & $0.015 \pm 0.011$ & $1.000 \pm 0.000$ \\
RealityMining & $\mathbf{0 . 7 7 8} \pm 0.111$ & $1.833 \pm 0.153$ & $\mathbf{0 . 2 9 5} \pm 0.126$ & $1.000 \pm 0.000$ \\
RemoteSensing & $\mathbf{1 . 2 0 1} \pm 0.053$ & $2.541 \pm 0.265$ & $\mathbf{0 . 5 2 9} \pm 0.094$ & $1.000 \pm 0.000$ \\
TW- $Y$ T-FF & $\mathbf{1 . 0 9 1} \pm 0.473$ & $2.660 \pm 0.121$ & $\mathbf{0 . 3 0 1} \pm 0.233$ & $0.917 \pm 0.144$ \\
\hline \hline \multicolumn{5}{|c|}{ ML-LCD-clsim } \\
\hline Airlines & $0.557 \pm 0.180$ & $2.208 \pm 0.485$ & $0.035 \pm 0.052$ & $0.277 \pm 0.252$ \\
AUCS & $1.249 \pm 0.221$ & $2.022 \pm 0.269$ & $0.447 \pm 0.237$ & $0.824 \pm 0.176$ \\
Biogrid & $0.336 \pm 0.332$ & $6.593 \pm 2.439$ & $0.037 \pm 0.044$ & $1.000 \pm 0.000$ \\
DBLP & $\mathbf{0 . 0 2 8} \pm 0.020$ & $2.306 \pm 0.324$ & $0.010 \pm 0.008$ & $1.000 \pm 0.000$ \\
RealityMining & $1.165 \pm 0.276$ & $2.743 \pm 0.287$ & $0.113 \pm 0.083$ & $0.719 \pm 0.286$ \\
RemoteSensing & $1.244 \pm 0.183$ & $3.073 \pm 0.379$ & $0.232 \pm 0.043$ & $1.000 \pm 0.000$ \\
TW - YT-FF & $1.211 \pm 0.955$ & $3.771 \pm 1.440$ & $0.062 \pm 0.058$ & $0.546 \pm 0.506$ \\
\hline
\end{tabular}

\subsubsection{Size of local communities}

Table 2 compares our ML-LCD methods in terms of size of the local communities produced. (We refer here only to ML-LCD methods, while results corresponding to LCD and Lemon will be discussed later in Sect. 5.2). On average, ML-LCD- $l w$ yields the largest communities on all datasets, except $T W$ - $Y T-F F$; on this dataset, which has a unique combination of highest $A_{d e g}$ and lowest $A_{\text {layer }}$ (cf. Table 1), ML-LCD-clsim communities slightly prevail in size w.r.t. ML-LCD-wlsim, though the latter shows the largest variation. In general, MLLCD-wlsim yields the smallest communities on all datasets (with the exception of RemoteSensing on which however it shows the smallest variation). Note also that, on $D B L P$, all methods tend to identify quite small communities, which can be explained due to the nature of the node relation (i.e., co-authorship). 
Table 4 Pairwise comparison of communities produced by ML-LCD methods: mean and standard deviation of Jaccard similarity. Bold values correspond to the highest per-dataset (mean) similarities.

\begin{tabular}{|l|c|c|c|}
\hline Dataset & $\begin{array}{c}\text { ML-LCD- } \text { lw vs. } \\
\text { ML-LCD-wlsim }\end{array}$ & $\begin{array}{c}\text { ML-LCD- } \text { wlsim vs. } \\
\text { ML-LCD-clsim }\end{array}$ & $\begin{array}{c}\text { ML-LCD-lw vs. } \\
\text { ML-LCD-clsim }\end{array}$ \\
\hline \hline Airlines & $0.345 \pm 0.351$ & $0.389 \pm 0.397$ & $\mathbf{0 . 3 9 1} \pm 0.349$ \\
AUCS & $0.641 \pm 0.314$ & $\mathbf{0 . 6 8 8} \pm 0.288$ & $0.638 \pm 0.364$ \\
Biogrid & $0.44 \pm 0.40$ & $\mathbf{0 . 7 2 2} \pm 0.376$ & $0.425 \pm 0.395$ \\
DBLP & $0.753 \pm 0.306$ & $\mathbf{0 . 8 0 7} \pm 0.258$ & $0.674 \pm 0.317$ \\
RealityMining & $0.536 \pm 0.361$ & $\mathbf{0 . 5 4 2} \pm 0.319$ & $0.458 \pm 0.308$ \\
RemoteSensing & $\mathbf{0 . 5 3 6} \pm 0.305$ & $0.531 \pm 0.234$ & $0.444 \pm 0.265$ \\
TW- $Y T-F F$ & $0.254 \pm 0.300$ & $\mathbf{0 . 3 2 8} \pm 0.325$ & $0.237 \pm 0.251$ \\
\hline
\end{tabular}

\subsubsection{Structure of the communities}

Table 3 reports the per-layer average path length and clustering coefficient of the identified communities. For each of the datasets, ML-LCD methods, and measures, we report mean and standard deviation over the layers, of the mean and maximum values found over all communities. ML-LCD- $l w$ communities tend to have the highest mean and maximum values of average path length, followed by ML-LCD-clsim. This is likely to be related to the different sizes of communities produced by the methods. Moreover, all methods' communities show very low average path length on $D B L P$, which again depends on the co-authorship type of relations.

Considering the clustering coefficient values, the three methods behave quite similarly to each other on $A U C S, D B L P$, and RealityMining. Some clique communities are identified on Biogrid, DBLP and RealityMining (all methods), $T W-Y T-F F$ (ML-LCD-lw), and RealityMining (ML-LCD-wlsim). Also, by coupling with average path length results, roughly small-world communities are observed on AUCS, RealityMining, RemoteSensing and (for ML-LCD-wlsim) $T W-Y T-F F$. The lowest values of average path length and clustering coefficient is achieved by all methods on $D B L P$, which might be explained due to the very sparse connectivity in this dataset (cf. Table 1).

\subsubsection{Community similarity}

We also compared our methods in terms of Jaccard similarity between the sets of nodes belonging to the local communities respectively extracted for the same seed node. Table 4 summarizes the mean and standard deviation similarities aggregated over all local communities.

The highest average similarity is generally achieved by ML-LCD-wlsim vs. ML-LCD-clsim, with marginal exceptions for Airlines (ML-LCD-lw vs. MLLCD-clsim) and RemoteSensing (ML-LCD-lw vs. ML-LCD-wlsim). Also, the comparison between ML-LCD-lw and ML-LCD-clsim always results in the lowest average similarity with the exception of Airlines where ML-LCD-lw and ML-LCD-wlsim behave more differently from each other. 


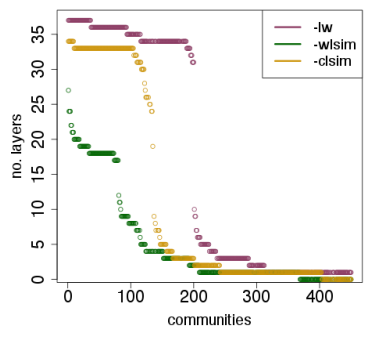

(a) Airlines

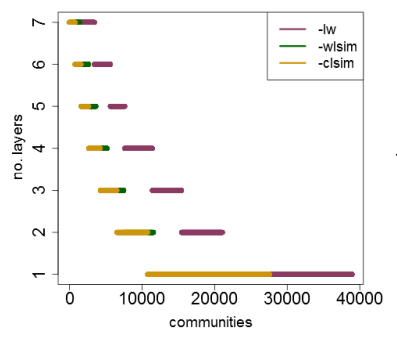

(b) Biogrid

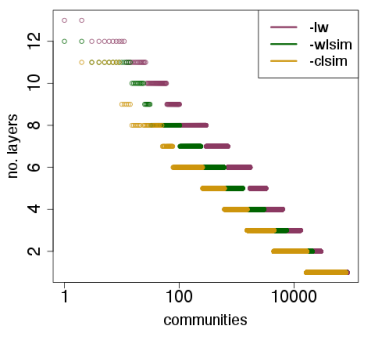

(c) $D B L P$

Fig. 2 Distribution of number of layers over communities. (Best viewed in color version, available in electronic format)

\subsubsection{Layer coverage over communities}

We analyzed the number of layers covered by each particular community, for each of the ML-LCD methods. Figure 2 reports results for the datasets with higher number of layers, i.e., Airlines, Biogrid and DBLP. (Communities are sorted by decreasing number of layers.) On $D B L P$, all methods show a stairslike behavior on the various layers, with a tendency of ML-LCD-clsim to produce more communities that cover a lower number of layers. A less regular form of stairs-like trend is observed for Biogrid, with a long tail (more evident for ML-LCD-clsim) of single-layer communities. More interestingly, on Airlines, a large fraction of communities (above $50 \%$ ) by ML-LCD-lw cover all or most of the layers; ML-LCD-clsim here also produces communities that lie on most layers, although for a much smaller fraction of them (about 12\%), whereas ML-LCD-wlsim produces about $25 \%$ of the communities covering $50-70 \%$ of layers.

Moreover, considering the datasets with the highest network-specific average ratio of layers per node (i.e., AUCS, RealityMining and RemoteSensing), we observe that all methods are able to produce most of the communities that cover all layers (results not shown). On $T W-Y T-F F$, at least two of three layers are always covered by a community.

\subsubsection{Distributions of communities for number of edges}

In addition to the previous analysis, we also studied the per-layer distribution of communities for number of edges. Due to space limitation, we report here details on AUCS and Airlines, which can be regarded as representatives of our evaluation datasets in terms of layer coverage and sparseness. Figures 34 show the kernel density estimate of the community distribution for number of edges, over each layer in a network.

On AUCS (Fig. 3), when using ML-LCD-lw, layers \#3 and \#4 characterize most of the communities with number of edges less than 10 and around 20, 


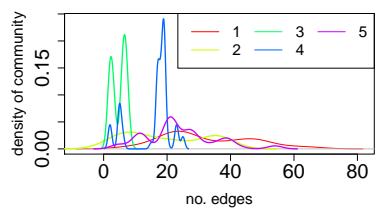

(a) ML-LCD-lw

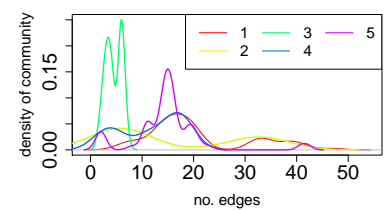

(b) ML-LCD-wlsim

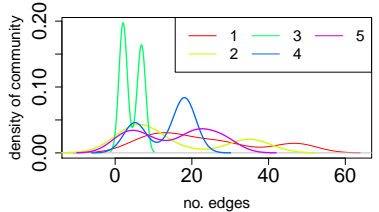

(c) ML-LCD-clsim

Fig. 3 Kernel density estimates of per-layer community distributions for number of edges, on AUCS. (Best viewed in color version, available in electronic format)

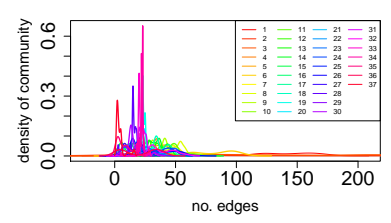

(a) ML-LCD-lw

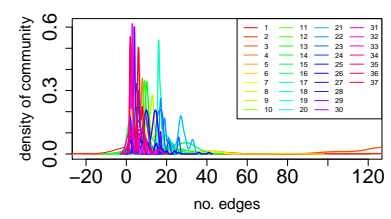

(b) ML-LCD-wlsim

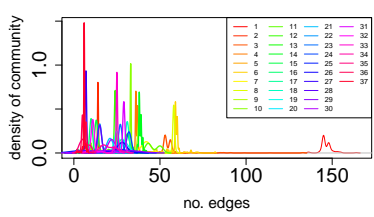

(c) ML-LCD-clsim

Fig. 4 Kernel density estimates of per-layer community distributions for number of edges, on Airlines. (Best viewed in color version, available in electronic format)

Table 5 Mean and standard deviation of the size of the communities identified by ML-LCDwlsim and ML-LCD-clsim using Cosine and triad-based similarity measures. Bold values correspond to the highest per-dataset (mean) size.

\begin{tabular}{|c|c|c|c|c|}
\hline \multirow{2}{*}{ Dataset } & \multicolumn{2}{|c|}{ ML-LCD- wlsim } & \multicolumn{2}{|c|}{ ML-LCD-clsim } \\
\hline & $\cos$ & triads & $\cos$ & triads \\
\hline Airlines & $13.348 \pm 17.023$ & $8.127 \pm 11.968$ & $\overline{\mathbf{2 1 . 3 6 2} \pm 22.197}$ & $19.273 \pm 21.324$ \\
\hline$A U C S$ & $8.869 \pm 3.032$ & $8.328 \pm 2.274$ & $\mathbf{1 2 . 2 3 0} \pm 8.538$ & $8.689 \pm 4.298$ \\
\hline Biogrid & $5.383 \pm 11.748$ & $4.953 \pm 3.919$ & $\mathbf{2 0 . 4 1 9} \pm 57.634$ & $7.338 \pm 16.300$ \\
\hline$D B L P$ & $3.266 \pm 2.466$ & $3.642 \pm 2.108$ & $3.706 \pm 3.930$ & $\mathbf{3 . 7 6 4} \pm 2.843$ \\
\hline RealityMin & $3.523 \pm 2.276$ & $3.333 \pm 1.805$ & $\mathbf{1 0 . 2 7 3} \pm 10.441$ & $7.632 \pm 5.859$ \\
\hline RemoteSensing & $5.865 \pm 2.620$ & $5.926 \pm 2.714$ & $\mathbf{7 . 9 4 5} \pm 4.276$ & $7.006 \pm 3.844$ \\
\hline$T W-Y T-F F$ & $\mathbf{2 5 . 7 8 5} \pm 42.462$ & $16.700 \pm 29.853$ & $18.209 \pm 17.020$ & $9.080 \pm 5.656$ \\
\hline
\end{tabular}

respectively. For the other layers there is instead a tendency to be covered by communities of varying size (in the full x-axis, for layer \#1). Considering ML-LCD-wlsim and ML-LCD-clsim, layer \#3 characterizes the majority of communities with smaller number of edges, while peaks of layer \#5 and \#4, respectively, correspond to less than 20 edges per community.

On Airlines (Fig. 4), distributions appear very right-skewed w.r.t. the number of edges. For ML-LCD-lw, the highest density corresponds to a small group of layers (being \#34 the dominant one), in the regime around 25 edges. Much more diversified is the situation for ML-LCD-wlsim and ML-LCD-clsim, where more layers are characteristic for a relatively large number of edges; in particular, ML-LCD-clsim is able to produce communities where more than five layers correspond to high density for a number of edges between ten and forty.

\subsubsection{Impact of similarity measures on $M L-L C D$-wlsim and $M L-L C D$-clsim}

We investigated the effect of using measures alternative to Jaccard similarity (jac), which is chosen as default in ML-LCD-wlsim and ML-LCD-clsim (cf. 
Table 6 Average Jaccard similarity of the node sets corresponding to the communities obtained by ML-LCD-wlsim and ML-LCD-clsim using Jaccard, Cosine and triad-based similarity measures. Mean and standard deviation values are reported. Bold values correspond to the highest per-dataset (mean) similarities.

\begin{tabular}{|l||ccc||ccc|}
\hline \multicolumn{1}{|l|}{ Dataset } & \multicolumn{3}{|c||}{ ML-LCD-wlsim } & \multicolumn{3}{c|}{ ML-LCD-clsim } \\
\cline { 2 - 7 } & jac vs. cos & jac vs. triads & cos vs. triads & jac vs. os & jac vs. triads & cos vs. triads \\
\hline Airlines & $0.712 \pm 0.286$ & $0.680 \pm 0.359$ & $0.554 \pm 0.328$ & $0.776 \pm 0.274$ & $\mathbf{0 . 8 3 6} \pm 0.291$ & $0.717 \pm 0.308$ \\
AUCS & $\mathbf{0 . 9 1 4} \pm 0.155$ & $0.861 \pm 0.227$ & $0.818 \pm 0.235$ & $0.804 \pm 0.237$ & $0.802 \pm 0.258$ & $0.716 \pm 0.246$ \\
Biogrid & $0.769 \pm 0.274$ & $0.680 \pm 0.349$ & $0.669 \pm 0.366$ & $0.664 \pm 0.345$ & $\mathbf{0 . 9 0 5} \pm 0.234$ & $0.650 \pm 0.229$ \\
DBLP & $0.774 \pm 0.221$ & $\mathbf{0 . 9 0 0} \pm 0.213$ & $0.725 \pm 0.239$ & $0.739 \pm 0.221$ & $0.899 \pm 0.214$ & $0.714 \pm 0.229$ \\
RealityMining & $0.873 \pm 0.218$ & $\mathbf{0 . 9 4 6} \pm 0.136$ & $0.839 \pm 0.237$ & $0.682 \pm 0.251$ & $0.745 \pm 0.255$ & $0.659 \pm 0.248$ \\
RemoteSensing & $\mathbf{0 . 8 7 2} \pm 0.219$ & $0.810 \pm 0.251$ & $0.809 \pm 0.246$ & $0.661 \pm 0.256$ & $0.660 \pm 0.301$ & $0.671 \pm 0.279$ \\
TW- $Y$ T-FF & $0.734 \pm 0.333$ & $\mathbf{0 . 8 2 4} \pm 0.231$ & $0.701 \pm 0.294$ & $0.683 \pm 0.272$ & $0.728 \pm 0.276$ & $0.625 \pm 0.292$ \\
\hline
\end{tabular}

Table 7 Percentage of identical communities obtained by ML-LCD-wlsim and ML-LCDclsim using Jaccard, Cosine and triad-based similarity measures. Bold values correspond to the highest per-dataset percentages.

\begin{tabular}{|l||ccc||ccc|}
\hline \multirow{2}{*}{ Dataset } & \multicolumn{3}{|c||}{ ML-LCD-wlsim } & \multicolumn{3}{c|}{ ML-LCD-clsim } \\
\cline { 2 - 6 } & jac vs. cos & jac vs. triads & cos vs. triads & jac vs. cos & jac vs. triads & cos vs. triads \\
\hline Airlines & $32.80 \%$ & $46.50 \%$ & $24.60 \%$ & $28.40 \%$ & $\mathbf{4 8 . 9 0 \%}$ & $23.90 \%$ \\
AUCS & $\mathbf{5 9 . 0 0 \%}$ & $55.70 \%$ & $34.40 \%$ & $36.10 \%$ & $52.50 \%$ & $26.20 \%$ \\
Biogrid & $\mathbf{5 0 . 7 0 \%}$ & $47.88 \%$ & $29.57 \%$ & $30.98 \%$ & $45.07 \%$ & $22.53 \%$ \\
DBLP & $41.50 \%$ & $76.10 \%$ & $33.60 \%$ & $32.00 \%$ & $\mathbf{7 6 . 6 0 \%}$ & $28.50 \%$ \\
RealityMining & $70.10 \%$ & $\mathbf{8 1 . 6 0 \%}$ & $63.20 \%$ & $23.00 \%$ & $39.10 \%$ & $21.80 \%$ \\
RemoteSensing & $\mathbf{6 5 . 1 0 \%}$ & $49.00 \%$ & $49.00 \%$ & $22.50 \%$ & $31.50 \%$ & $26.30 \%$ \\
TW-YT-FF & $27.31 \%$ & $38.76 \%$ & $25.10 \%$ & $23.69 \%$ & $\mathbf{4 0 . 7 6 \%}$ & $22.00 \%$ \\
\hline
\end{tabular}

Section 4.3p. Specifically, we discuss results concerning the size, similarity and matching of communities produced by the two methods when equipped with each of the similarity measures.

Looking at Table 5, we observe that the average size of the communities obtained using cosine similarity or triad-based similarity is, in many cases, higher than the one obtained using Jaccard (cf. Table 2), for both ML-LCD methods. One exception is represented by $D B L P$, where all the methods obtain comparable average sizes. This is probably due to the low connectivity and the high number of cliques this dataset contains; moreover, in $D B L P$, almost the same average community size is obtained by using triads and jac. As a side remark, the method used (i.e., ML-LCD-wlsim or ML-LCD-clsim) does not seem to have a strong bias on the relative differences among the behaviors of the different similarity measures, for what concerns the size of the communities.

Table 6 reports the average Jaccard similarities between the node sets of the communities obtained by ML-LCD-wlsim and ML-LCD-clsim, by varying the similarity measures. It can be noted that the most different communities (i.e., the lowest Jaccard similarity values) are obtained when comparing communities detected by cos and triads, for both ML-LCD-wlsim and MLLCD-clsim (with the only exception of ML-LCD-clsim on RemoteSensing). As regards the most similar communities, on ML-LCD-clsim higher similarity values are generally achieved comparing $j a c$ and triads measures. Even when this comparison does not correspond to the highest similarity of community, i.e., on AUCS and RemoteSensing, it is very close to the maximum value, which is therein obtained by comparing $j a c$ and $c o s$. This is not surprising since jac and triads are both Jaccard-based measures, and on some specific networks 
Table 8 Community structure overlap: mean and standard deviation of the Jaccard coefficients of the node sets over all pairs of local communities generated by each of the ML-LCD methods. Bold values correspond to the highest per-dataset (mean) community overlaps.

\begin{tabular}{|l||c|c|c|c|c|c|c|}
\hline Dataset & Airlines & AUCS & Biogrid & DBLP & RealityMining & RemoteSensing & TW-YT-FF \\
\hline \hline lw & $\mathbf{0 . 1 3 6} \pm 0.252$ & $\mathbf{0 . 1 8 4} \pm 0.347$ & $0.001 \pm 0.018$ & $0 \pm 0.002$ & $0.080 \pm 0.203$ & $\mathbf{0 . 0 1 1} \pm 0.081$ & $0.003 \pm 0.030$ \\
wlsim_jac & $0.037 \pm 0.155$ & $0.122 \pm 0.310$ & $0.000 \pm 0.012$ & $0 \pm 0.002$ & $0.040 \pm 0.176$ & $0.008 \pm 0.077$ & $0.004 \pm 0.050$ \\
wlsim_cos & $0.040 \pm 0.153$ & $0.142 \pm 0.323$ & $0.000 \pm 0.012$ & $0 \pm 0$ & $0.039 \pm 0.175$ & $0.009 \pm 0.080$ & $0.004 \pm 0.055$ \\
wlsim_triads & $0.019 \pm 0.102$ & $0.131 \pm 0.321$ & $0.009 \pm 0.079$ & $0 \pm 0.002$ & $0.035 \pm 0.160$ & $0.009 \pm 0.079$ & $0.003 \pm 0.056$ \\
clsim_jac & $0.090 \pm 0.260$ & $0.135 \pm 0.307$ & $0.000 \pm 0.010$ & $0 \pm 0.002$ & $0.058 \pm 0.167$ & $0.007 \pm 0.064$ & $0.008 \pm 0.071$ \\
clsim_cos & $0.092 \pm 0.268$ & $0.158 \pm 0.317$ & $0.001 \pm 0.025$ & $0 \pm 0$ & $\mathbf{0 . 0 8 7} \pm 0.207$ & $0.010 \pm 0.077$ & $0.008 \pm 0.066$ \\
clsim_triads & $0.091 \pm 0.261$ & $0.125 \pm 0.301$ & $\mathbf{0 . 0 1 4} \pm 0.084$ & $0 \pm 0.002$ & $0.066 \pm 0.173$ & $0.009 \pm 0.071$ & $\mathbf{0 . 0 1 2} \pm 0.089$ \\
\hline
\end{tabular}

the number of shared 3-cliques can be very similar to the number of shared neighbors, making the effects due to these two measures close to each other. As regards ML-LCD-wlsim, the highest similarity is achieved again when comparing $j a c$ and triads on DBLP, RemoteSensing and $T W-Y T-F F$, and when comparing $j a c$ and $c o s$ on the other datasets. The latter would indicate that in the rest of the datasets the neighborhood of nodes to be compared tend to be of comparable sizes.

We further studied the effect of alternative measures by observing the percentage of identical communities obtained by ML-LCD-wlsim and ML-LCDclsim. Looking at Table 7, this ranges from $24.60 \%$ to $81.60 \%$ for ML-LCDwlsim, and from $21.80 \%$ to $76.60 \%$ for ML-LCD-clsim. Generally, high percentages are achieved on $D B L P$, which again is explained by the particular structure of this network. Considering ML-LCD-clsim, this appears to be more sensitive to the choice of similarity measure since the percentage of identical communities is general lower that the corresponding values obtained by ML-LCD-wlsim. In both cases, highest values are achieved considering the comparison between $j a c$ and triads measures. As regards ML-LCD-wlsim, relatively high percentages are obtained on RemoteSensing and RealityMining, i.e., datasets which are relatively small in size and number of layers. Conversely, the fraction of identical communities decreases considering networks with more layers and higher connectivity (e.g., on Airlines).

\subsubsection{Community structure overlap}

In this analysis we evaluated the amount of overlap among the communities identified by each of the ML-LCD methods on the various networks. To this purpose, we computed the Jaccard coefficients of the node sets over all pairs of local communities identified by any particular ML-LCD method. Results, shown in Table 8 , indicate that each of the methods, on all datasets, produces local communities sharing, on average, relatively few nodes with the other communities in the network. In all cases, with the exception of all methods on AUCS and ML-LCD-lw on Airlines, the mean Jaccard coefficient is below 0.1 , while the standard deviations are generally higher than the corresponding means, up to 0.3 on $A U C S$.

This might be explained considering the network characteristics reported in Table 1, and the semantics of our evaluation data (as described in Section 4.1). 
From a structural point of view, networks on which ML-LCD methods generate communities with more overlap are relatively smaller in terms of nodes (AUCS, Airlines and RealityMining), while less overlapping communities are identified for sparser and larger networks (RemoteSensing, Biogrid and $T W$ $Y T-F F)$. The extreme case is represented by the $D B L P$ network where the extracted communities roughly form a partition of the network. We remind that this network contains many small connected components and it is characterized by high sparsity and the lowest average degree per node ( $A_{d e g}$ equals to 3.8). The slightly higher overlap of community structure observed on smaller datasets (i.e., AUCS, Airlines and RealityMining) also finds an intuitive explanation in the nature of the multilayer data, and in particular in the presence of hubs or bridges in the network. For instance, in the flight routing scenario corresponding to Airlines, some nodes may represent airport locations that are main hubs in a certain geographical area (e.g., major European capital cities). These hubs would be connected to the majority of smaller airports in the area, and with other main hubs, for almost all airline companies flying in that area, therefore they will be more likely to be included in several local communities. As concerns AUCS and RealityMining, since these networks represent different online/offline interactions between (relatively) small sets of individuals, some highly popular individuals may act as "bridges" across layers, causing a certain overlap in the local communities built around them and their neighbors. For instance, the chief of the department represented in AUCS is likely to appear, together with full professors, in several local communities. A similar scenario can be figured out for RealityMining where members of the MIT Media Laboratory and members of the MIT Sloan business school can belong to different communities since they attend the same master classes and/or sport activities in the campus.

\subsubsection{Efficiency analysis}

We discuss here the time performances of ML-LCD. Table 9 shows, for each dataset and method, the average, standard deviation and maximum execution times, over all nodes in the network. Note that minimum time values are not reported, since they are of the order of magnitude of $1.0 \mathrm{e}-4$ in all cases, except for $T W-Y T-F F$ where they correspond to few seconds for all methods.

Considering first ML-LCD-lw and the default versions of ML-LCD-wlsim and ML-LCD-clsim ( $j a c$ option), it can be noted that, when the methods produce communities of comparable size (like in AUCS, DBLP and RemoteSensing; cf. Table 2), ML-LCD-lw and ML-LCD-wlsim have similar time performance while ML-LCD-clsim tends to be slower; in particular, on $D B L P$, this behavior appears to be more evident considering the maximum time, since the average time is biased by the very sparse connectivity of this network and the presence of many, small, connected components in which the size of the shell set plays a minor role than in the other networks. Conversely, on Biogrid and RealityMining, ML-LCD-lw tends to run more slowly than the other two, whereas on Airlines the time performances of ML-LCD-lw are between those of 
Table 9 Time performance (in seconds) of ML-LCD- lw, ML-LCD-wlsim and ML-LCD-clsim using different similarity measures. Mean, standard deviation and maximum values are computed over the execution times of each ML-LCD method over all nodes in each network, by selecting one node at a time as seed. Bold values and underlined values correspond to the lowest and the highest mean time, respectively.

\begin{tabular}{|c|c|c|c|c|c|c|c|c|c|c|c|c|c|c|}
\hline \multirow[t]{2}{*}{ Dataset } & \multicolumn{2}{|c|}{$l w$} & \multicolumn{2}{|c|}{ wlsim_jac } & \multicolumn{2}{|c|}{ wlsim_cos } & \multicolumn{2}{|c|}{ wlsim_triads } & \multicolumn{2}{|c|}{ clsim_jac } & \multicolumn{2}{|c|}{ clsim_cos } & \multicolumn{2}{|c|}{ clsim_triads } \\
\hline & $\begin{array}{c}\text { mean } \\
\text { sd }\end{array}$ & & $\begin{array}{c}\text { mean } \\
\text { sd }\end{array}$ & $\operatorname{ax}$ & $\begin{array}{c}\text { mean } \\
\text { sd }\end{array}$ & & $\begin{array}{c}\text { mean } \\
\text { sd }\end{array}$ & ax & $\begin{array}{c}\text { mean } \\
\text { sd }\end{array}$ & $\mathrm{ax}$ & $\begin{array}{c}\text { mean } \\
\text { sd }\end{array}$ & & $\begin{array}{c}\text { mean } \\
\text { sd }\end{array}$ & $\operatorname{ax}$ \\
\hline Airl & \begin{tabular}{|l|}
3.872 \\
4.342 \\
\end{tabular} & & \begin{tabular}{|l|}
$\mathbf{1 . 3 5 1}$ \\
2.393
\end{tabular} & & \begin{tabular}{|l|}
2.184 \\
3.479
\end{tabular} & & & & \begin{tabular}{|c|}
7.252 \\
10.182
\end{tabular} & & & & $\frac{236}{319}$ & 824 \\
\hline$A U$ & $\begin{array}{l}42 \\
42 \\
\end{array}$ & ( & $\begin{array}{c}\mathbf{0 . 0 4 2} \\
0.036 \\
\end{array}$ & 4 & \begin{tabular}{|l|l|}
0.065 \\
0.052 \\
\end{tabular} & c & & 1.258 & & 4 & & 2.832 & $\frac{1.451}{1.676}$ & 7.9 \\
\hline Biog & \begin{tabular}{|c|}
68.8 \\
1196 \\
\end{tabular} & 61789 & $\begin{array}{l}13.5 \\
106 \\
\end{array}$ & 5188 & \begin{tabular}{|l|}
$\mathbf{1 0 . 2}$ \\
65.9 \\
\end{tabular} & 598 & $\frac{1670}{7840}$ & $27 \varepsilon$ & $\begin{array}{l}43.2 \\
637\end{array}$ & 88 & $\begin{array}{l}42.1 \\
490\end{array}$ & 837 & \begin{tabular}{|l|}
1073 \\
5348 \\
\end{tabular} & 633 \\
\hline$D B L P$ & \begin{tabular}{|l|}
0.005 \\
0.020 \\
\end{tabular} & 0.339 & $\begin{array}{l}\mathbf{0 . 0 0 4} \\
0.011\end{array}$ & ( & \begin{tabular}{|l|}
0.006 \\
0.022
\end{tabular} & 0 & $\begin{array}{l}0.115 \\
0.607\end{array}$ & 13.329 & $\begin{array}{l}0.005 \\
0.037\end{array}$ & 1.179 & $\begin{array}{l}0.016 \\
0.139 \\
\end{array}$ & 3.826 & $\frac{0.137}{1.504}$ & \\
\hline Rea & \begin{tabular}{|l|}
0.049 \\
0.087
\end{tabular} & 0.317 & $\begin{array}{c}\mathbf{0 . 0 1 0} \\
0.010\end{array}$ & 0.045 & \begin{tabular}{|l|}
0.011 \\
0.011
\end{tabular} & c & 0.467 & 1.800 & & 0.214 & $\begin{array}{l}0.090 \\
0.163\end{array}$ & 0.845 & \begin{tabular}{|l|}
$\frac{0.678}{0.832}$ \\
\end{tabular} & 3.395 \\
\hline RemoteSensing & \begin{tabular}{|l|}
$\mathbf{0 . 0 1 9}$ \\
0.024 \\
\end{tabular} & 0. & $\begin{array}{l}0.021 \\
0.028 \\
\end{array}$ & 0.265 & \begin{tabular}{|l|}
0.022 \\
0.018 \\
\end{tabular} & $0 .($ & $\begin{array}{l}0.064 \\
0.059 \\
\end{array}$ & 0.480 & \begin{tabular}{|l|}
0.038 \\
0.058 \\
\end{tabular} & 364 & $\begin{array}{l}0.094 \\
0.126 \\
\end{array}$ & 776 & $\frac{0.213}{0.337}$ & 2.6 \\
\hline$T W-Y T-F F$ & $\begin{array}{c}601 \\
1682 \\
\end{array}$ & 9 & $\begin{array}{l}123 \\
578 \\
\end{array}$ & 3 & \begin{tabular}{|l|}
1493 \\
2270 \\
\end{tabular} & 62 & $\frac{133514}{105151}$ & & \begin{tabular}{|l|}
13493 \\
38922 \\
\end{tabular} & & $\begin{array}{l}100 \\
239\end{array}$ & 856 & $\begin{array}{l}11952 \\
13190\end{array}$ & \\
\hline
\end{tabular}

ML-LCD-wlsim and ML-LCD-clsim; also, on TW-YT-FF, ML-LCD-clsim can perform better than ML-LCD-wlsim. The above findings are clearly related to both the computational complexity of each of the ML-LCD methods as well as to the size of the communities extracted by the methods; for instance, on Airlines, Biogrid and RealityMining, ML-LCD-lw communities are bigger (from two to four times) than those extracted by the other methods; also, on $T W-Y T-F F$, the size obtained by ML-LCD-wlsim with triads option is nearly double the size of ML-LCD-clsim communities (Table 5).

It should be noted that the time performance of the various methods on $T W-Y T-F F$ is several orders of magnitude higher than in the other datasets (up to an average execution time of 133514 seconds for wlsim_triads and absolute maximum time for clsim_jac). This is explained since, as reported in Table 1] $T W-Y T-F F$ is the dataset having the highest average degree of node and, among the largest datasets, is also the densest one (one order of magnitude denser than Biogrid and $D B L P)$. These characteristics make $T W$ $Y T-F F$ our hardest benchmark in terms of time performance obtained by our methods.

Concerning the impact of the different similarity measures on the time performances, the execution time of the methods tend to become much slower when triads is used, given the generally higher cost of computing all the 3cliques in which a node is involved than analyzing its neighbors. Moreover, options jac and cos generally lead ML-LCD-wlsim and ML-LCD-clsim to behave similarly (which is quite expected since the two similarity measures have the same cost), in all cases with the exception of $T W-Y T-F F$ - for the characteristics of this dataset above discussed, the Jaccard measure could lead to particularly slow performance, as it may yield high values (i.e., the nodes may share a large portion of their neighborhood), thus being quite inclusive when building a local community. 


\subsubsection{Discussion}

We summarize here the main results from the evaluation of our ML-LCD methods. First, ML-LCD-lw followed by ML-LCD-clsim have shown to produce larger communities on all datasets. This indicates that in general the approach underlying ML-LCD-lw (i.e., fixed layer-based weighting scheme) tends to be less "xenophobic" than ML-LCD-clsim, and this in turn less than MLLCD-wlsim. This is actually explained since the impact of the $L C^{e x t}$ term is generally smaller for the ML-LCD- $l w$ formulation allowing it to grow more the local community for a particular seed node. A different situation corresponds to ML-LCD-clsim where the $L C^{e x t}$ function is computed between the boundary nodes and all their neighbors without considering the layers in which they lie.

Related to the above aspect is that ML-LCD-lw followed by ML-LCD-clsim provide communities having the highest mean and maximum values of average path length. Moreover, all methods are able to produce roughly small-world communities (on AUCS, RealityMining, and ML-LCD-wlsim on TW-YT-FF).

ML-LCD-wlsim and ML-LCD-clsim are more likely to behave similarly, in terms of Jaccard similarity between the extracted communities for the same node. This is not surprising, since these two formulations are based on a similar notion of node similarity, while differing in how layer features are considered. Moreover, for both methods, cosine similarity and triad-based similarity show a generally more aggregating behavior than the default Jaccard similarity in most cases.

ML-LCD-lw and ML-LCD-clsim generally produce communities that cover all or most of the layers. This holds consistently with various distributions of the number of layers per node; when the latter is quite high (e.g., above $80 \%$ ), all ML-LCD methods are equally able to mostly produce communities that cover all or most of the layers.

Each of the ML-LCD methods produces local communities sharing, on average, relatively few nodes with the other communities identified in the same network dataset.

As concerns the efficiency of the proposed ML-LCD methods, the best performance is obtained by ML-LCD-wlsim (with Jaccard or cosine similarity) in most cases. Yet, this can be explained since, despite the ML-LCD-lw has the lowest computational complexity, it tends to discover larger local communities than the other methods, thus affecting the number of iterations to terminate. This efficiency result would make ML-LCD-wlsim a preferred choice for the ML-LCD problem, supporting our initial intuition on beneficial effects that might be obtained by incorporating node similarity over the layers into the objective function of the ML-LCD problem. 
Table 10 Comparison of ML-LCD methods with LCD: mean and standard deviation of Jaccard similarity of communities and percentage of identical communities (\%idc). Bold values correspond to the highest per-dataset (mean) similarities and percentages.

\begin{tabular}{|c|c|c|c|c|c|c|c|c|c|c|c|c|c|c|}
\hline \multirow[t]{2}{*}{ Dataset } & \multicolumn{2}{|r|}{$l w$} & \multicolumn{2}{|c|}{ wlsim_jac } & \multicolumn{2}{|c|}{ wlsim_cos } & \multicolumn{2}{|c|}{ wlsim_triads } & \multicolumn{2}{|c|}{ clsim_jac } & \multicolumn{2}{|c|}{ clsim_cos } & \multicolumn{2}{|c|}{ clsim_triads } \\
\hline & $\begin{array}{c}\text { mean } \\
\text { sd }\end{array}$ & \%idc & $\begin{array}{c}\text { mean } \\
\text { sd }\end{array}$ & \%idc & $\begin{array}{c}\text { mean } \\
\text { sd }\end{array}$ & \%idc & $\begin{array}{c}\text { mean } \\
\text { sd }\end{array}$ & \%idc & $\begin{array}{c}\text { mean } \\
\text { sd }\end{array}$ & \%idc & $\begin{array}{c}\text { mean } \\
\text { sd }\end{array}$ & \%idc & $\begin{array}{c}\text { mean } \\
\text { sd }\end{array}$ & \%idc \\
\hline Airli & \begin{tabular}{|l|}
0.28 \\
0.25
\end{tabular} & & \begin{tabular}{|l|}
0.27 \\
0.22
\end{tabular} & & $\begin{array}{l}\mathbf{0 . 3 6} \\
0.35\end{array}$ & & \begin{tabular}{|l|}
0.29 \\
0.23
\end{tabular} & & \begin{tabular}{|l|}
0.26 \\
0.23 \\
\end{tabular} & & \begin{tabular}{|l|}
0.34 \\
0.36
\end{tabular} & & \begin{tabular}{|l}
0.26 \\
0.23
\end{tabular} & \\
\hline$A U C$ & $\begin{array}{l}46 \\
32\end{array}$ & 4. & \begin{tabular}{|l|}
0.41 \\
0.31
\end{tabular} & 4. & \begin{tabular}{|l|l}
0.42 \\
0.32
\end{tabular} & $4.92 \%$ & \begin{tabular}{|l|}
0.43 \\
0.31
\end{tabular} & $\%$ & \begin{tabular}{|l|}
0.41 \\
0.30
\end{tabular} & 0. & \begin{tabular}{|l|}
0.42 \\
0.30
\end{tabular} & $\%$ & \begin{tabular}{|l|}
0.39 \\
0.29
\end{tabular} & $0.00 \%$ \\
\hline Biog & \begin{tabular}{|l|}
0.34 \\
0.25
\end{tabular} & & \begin{tabular}{|l|}
0.31 \\
0.20
\end{tabular} & & \begin{tabular}{|c|}
$\mathbf{0 . 4 4}$ \\
0.37
\end{tabular} & & \begin{tabular}{|l|}
0.23 \\
0.17
\end{tabular} & & \begin{tabular}{|l|}
0.29 \\
0.19
\end{tabular} & $\%$ & \begin{tabular}{|l|}
0.26 \\
0.30
\end{tabular} & & \begin{tabular}{|l|}
0.36 \\
0.18 \\
\end{tabular} & \\
\hline & \begin{tabular}{|l|}
0.59 \\
0.23
\end{tabular} & 10.7 & \begin{tabular}{|l|l}
0.55 \\
0.23
\end{tabular} & 6.08 & \begin{tabular}{|c|}
$\mathbf{0 . 6 9}$ \\
0.32
\end{tabular} & $41.20 \%$ & \begin{tabular}{|l|}
0.55 \\
0.22
\end{tabular} & $6.04 \%$ & \begin{tabular}{|l|l}
0.53 \\
0.21
\end{tabular} & $3.70 \%$ & \begin{tabular}{|l|}
0.65 \\
0.31
\end{tabular} & $36.40 \%$ & \begin{tabular}{|l}
0.53 \\
0.21
\end{tabular} & $.80 \%$ \\
\hline Reali & \begin{tabular}{|l|}
0.41 \\
0.24
\end{tabular} & 2. & \begin{tabular}{|l}
0.40 \\
0.22
\end{tabular} & $\%$ & \begin{tabular}{|c|}
$\mathbf{0 . 4 6}$ \\
0.30
\end{tabular} & & \begin{tabular}{|l|}
0.40 \\
0.21
\end{tabular} & 1. & \begin{tabular}{|l|}
0.35 \\
0.19
\end{tabular} & $0.00 \%$ & \begin{tabular}{|l|}
0.40 \\
0.30
\end{tabular} & $14.94 \%$ & \begin{tabular}{|l|}
0.34 \\
0.18
\end{tabular} & $0.00 \%$ \\
\hline$R$ & \begin{tabular}{|l|}
0.39 \\
0.21
\end{tabular} & 1.1 & \begin{tabular}{|l|}
0.39 \\
0.22
\end{tabular} & 1.2 & \begin{tabular}{|l|}
$\mathbf{0 . 4 1}$ \\
0.25
\end{tabular} & & \begin{tabular}{|l}
0.38 \\
0.20
\end{tabular} & 0 & \begin{tabular}{|l|}
0.34 \\
0.17
\end{tabular} & $0.00 \%$ & \begin{tabular}{|l|}
0.38 \\
0.22
\end{tabular} & $4.85 \%$ & \begin{tabular}{|l|}
0.33 \\
0.16
\end{tabular} & $.00 \%$ \\
\hline$T \mathrm{I}$ & \begin{tabular}{|l|}
0.19 \\
0.20 \\
\end{tabular} & $0.00 \%$ & \begin{tabular}{|l}
0.20 \\
0.20 \\
\end{tabular} & 0.0370 & \begin{tabular}{|l|}
0.24 \\
0.21 \\
\end{tabular} & 3.0970 & \begin{tabular}{|l|}
0.16 \\
0.34 \\
\end{tabular} & $000 \%$ & \begin{tabular}{|l|}
0.21 \\
0.20 \\
\end{tabular} & 1 & \begin{tabular}{|l|}
0.25 \\
0.22 \\
\end{tabular} & $\%$ & \begin{tabular}{|l|}
$\mathbf{0 . 3 1}$ \\
0.32 \\
\end{tabular} & \\
\hline
\end{tabular}

Table 11 Comparison of ML-LCD methods with Lemon: mean and standard deviation of Jaccard similarity of communities and percentage of identical communities (\%idc). Bold values correspond to the highest per-dataset (mean) similarities and percentages.

\begin{tabular}{|c|c|c|c|c|c|c|c|c|c|c|c|c|}
\hline \multirow[t]{2}{*}{ Dataset } & $l w$ & \multicolumn{2}{|c|}{$\overline{\text { wlsim_jac }}$} & \multicolumn{2}{|c|}{ wlsim_cos } & \multicolumn{2}{|c|}{ wlsim_triads } & \multicolumn{2}{|c|}{ clsim_jac } & \multicolumn{2}{|c|}{ clsim_cos } & clsim_triads \\
\hline & $\begin{array}{c}\text { mean } \\
\text { sd }\end{array} \% i d c$ & $\begin{array}{c}\text { mean } \\
\text { sd }\end{array}$ & $\% i d c$ & $\begin{array}{c}\text { mean } \\
\text { sd }\end{array}$ & \%idc & $\begin{array}{c}\text { mean } \\
\text { sd }\end{array}$ & $i d c$ & $\begin{array}{c}\text { mean } \\
\text { sd }\end{array}$ & $\% i d c$ & $\begin{array}{c}\text { mean } \\
\text { sd }\end{array}$ & $\%$ \%idc & $\begin{array}{c}\text { mean } \\
\text { sd }\end{array}$ \%idc \\
\hline Airli & \begin{tabular}{|ll}
$\mathbf{0 . 2 7}$ & 0.00 \\
0.23 &
\end{tabular} & \begin{tabular}{|l|}
0.12 \\
0.14
\end{tabular} & $\overline{\mathrm{C} \Omega 0}$ & \begin{tabular}{|l|}
0.14 \\
0.16
\end{tabular} & $\overline{0}$ & \begin{tabular}{|l|}
0.09 \\
0.11
\end{tabular} & $\%$ & $\begin{array}{l}0.18 \\
0.18\end{array}$ & $0.00 \%$ & \begin{tabular}{|l|}
0.18 \\
0.18
\end{tabular} & $0.00 \%$ & \begin{tabular}{|ll}
0.17 & $0.00 \%$ \\
0.18 &
\end{tabular} \\
\hline$A U C$ & $\begin{array}{|ll|}0.21 & 0.0 \\
0.08 & \\
\end{array}$ & \begin{tabular}{|l|}
0.15 \\
0.06
\end{tabular} & $0.00 \%$ & \begin{tabular}{|l|}
$\mathbf{0 . 4 2}$ \\
0.07
\end{tabular} & 0. & \begin{tabular}{|l|}
0.16 \\
0.06
\end{tabular} & & $\begin{array}{l}0.17 \\
0.08\end{array}$ & $\%$ & \begin{tabular}{|l|}
0.22 \\
0.13
\end{tabular} & & $\begin{array}{|ll|}0.16 & 0.00 \% \\
0.10 & \end{array}$ \\
\hline Biogrid & $\begin{array}{l}\mathbf{0 . 0 9} \\
0.10\end{array}$ & $\begin{array}{l}0.07 \\
0.08\end{array}$ & $\%$ & \begin{tabular}{|l|}
0.07 \\
0.09
\end{tabular} & $\%$ & .09 & $\%$ & $\begin{array}{l}0.07 \\
0.09\end{array}$ & $\%$ & \begin{tabular}{|l|}
$\mathbf{0 . 0 9}$ \\
0.12
\end{tabular} & $\%$ & \begin{tabular}{|ll}
0.06 & $0.00 \%$ \\
0.06 &
\end{tabular} \\
\hline$D B L P$ & \begin{tabular}{|ll}
$\mathbf{0 . 2 1}$ & 4.25 \\
0.22 &
\end{tabular} & \begin{tabular}{|l|}
0.20 \\
0.24
\end{tabular} & 5.1 & \begin{tabular}{|l|}
0.19 \\
0.24
\end{tabular} & $4.23 \%$ & $\begin{array}{l}0.20 \\
0.24\end{array}$ & & $\begin{array}{l}0.20 \\
0.24\end{array}$ & $\%$ & $\begin{array}{l}0.21 \\
0.24\end{array}$ & $\%$ & \begin{tabular}{|l|}
0.20 \\
0.24
\end{tabular} \\
\hline RealityMining & \begin{tabular}{|ll}
0.16 & $0.00 \%$ \\
0.14 & \\
\end{tabular} & $\begin{array}{l}0.08 \\
0.06\end{array}$ & $0.00 \%$ & \begin{tabular}{|l|}
0.09 \\
0.07
\end{tabular} & $000 \%$ & \begin{tabular}{|l|}
0.08 \\
0.06
\end{tabular} & $0.00 \%$ & $\begin{array}{l}0.16 \\
0.14\end{array}$ & $0.00 \%$ & \begin{tabular}{|l|}
$\mathbf{0 . 2 1}$ \\
0.18
\end{tabular} & $0.00 \%$ & \begin{tabular}{|ll}
0.18 & $0.00 \%$ \\
0.16 & \\
\end{tabular} \\
\hline Rem & $\begin{array}{ll}0.22 & 0.00 \% \\
0.11 & \end{array}$ & $\begin{array}{l}0.18 \\
0.09\end{array}$ & $0.00 \%$ & \begin{tabular}{|l|}
0.18 \\
0.10
\end{tabular} & $0.00 \%$ & 0.18 & $0.00 \%$ & $\begin{array}{l}0.17 \\
0.10\end{array}$ & $0.00 \%$ & \begin{tabular}{|l|}
$\mathbf{0 . 2 3}$ \\
0.13
\end{tabular} & $0.00 \%$ & $\begin{array}{|ll|}0.20 & 0.00 \% \\
0.12 & \end{array}$ \\
\hline & $\begin{array}{|ll|}0.07 & 0.03 \% \\
0.09 & \\
\end{array}$ & $\begin{array}{l}0.08 \\
0.12\end{array}$ & $\overline{0.08}$ & \begin{tabular}{|l|}
0.08 \\
0.13 \\
\end{tabular} & 0 & \begin{tabular}{|l|}
0.04 \\
0.04 \\
\end{tabular} & $00 \%$ & $\begin{array}{l}\mathbf{0 . 1 1} \\
0.12 \\
\end{array}$ & $\%$ & \begin{tabular}{|l|}
$\mathbf{0 . 1 1}$ \\
0.13 \\
\end{tabular} & $F_{0}$ & \begin{tabular}{|l|}
0.07 \\
0.09
\end{tabular} \\
\hline
\end{tabular}

5.2 Comparison with local community detection methods on the aggregate graphs

In this evaluation stage, our goal was to assess the difference in size and in composition between the local communities produced by ML-LCD and the ones (corresponding to the same seeds) produced by the LCD and Lemon methods (cf. Section 4.2).

Size values of the communities identified by LCD and Lemon are reported in Table 2, along with values corresponding to the ML-LCD methods. From the comparison, we observe that the mean size of communities by LCD are generally lower than the size of at least one of the ML-LCD methods; on AUCS and RemoteSensing, where LCD yields the largest communities on average, the corresponding values are however very close to those by ML-LCD-lw, yet with higher standard deviation. Different situation concerns Lemon, which in absence of ground-truth information on the communities to be identified (as in our case), tends to generate communities with significantly larger size than 
Table 12 Number of communities identified by LocToGlob (mean and standard deviation) and competing methods. Results of LocToGlob are aggregated over fifty runs. Bold values and underlined values correspond to the highest per-dataset community numbers considering ML-LCD methods and all methods, respectively.

\begin{tabular}{|l|c|c|c|c|c|}
\hline Dataset & LocToGlob-lw & LocToGlob-wlsim & LocToGlob-clsim & LART & GL \\
\hline \hline Airlines & $50.38 \pm 4.28$ & $\mathbf{6 7 . 3 6} \pm 5.18$ & $56.20 \pm 6.14$ & $\underline{381}$ & 12 \\
AUCS & $8.46 \pm 1.17$ & $\mathbf{1 0 . 2 4} \pm 1.11$ & $8.66 \pm 1.05$ & $\underline{\underline{27}}$ & 6 \\
Biogrid & $5959.0 \pm 105.53$ & $\mathbf{7 3 2 3 . 6 0} \pm 229.32$ & $6037.20 \pm 272.1$ & $\underline{\mathrm{NA}}$ & $\underline{16151}$ \\
RemoteSensing & $116.44 \pm 5.28$ & $134.76 \pm 5.10$ & $\mathbf{1 5 2 . 6 2} \pm 5.32$ & $\underline{218}$ & 19 \\
RealityMining & $20.64 \pm 2.30$ & $\mathbf{2 3 . 8 2} \pm 2.02$ & $20.42 \pm 2.55$ & $\underline{62}$ & 13 \\
TW-YT-FF & $1076.44 \pm 25.17$ & $\underline{\mathbf{1 2 5 5 . 7 1}} \pm 38.33$ & $1230.71 \pm 45.33$ & $\underline{\mathrm{NA}}$ & 761 \\
\hline
\end{tabular}

approaches based on the optimization of the internal-to-external connection density ratio.

Tables 1011 summarize results relating to the comparison with LCD and Lemon, respectively, in terms of Jaccard similarity (mean and standard deviation values over all seed nodes) and fraction of identical communities (i.e., Jaccard similarity equal to one). Considering Table 10 and looking at the mean similarity values w.r.t. $\mathrm{LCD}$, it appears that on all datasets, but $D B L P$, they are always below 0.5 , with maximum values per dataset that are reached when the cos option is used. Also, the percentage of identical communities w.r.t. LCD is generally very low or even null (again, with the exception of $D B L P$ where ML-LCD-wlsim and ML-LCD-clsim equipped with cos obtain about $40 \%$ of same communities as those by LCD); clearly, the situation observed for $D B L P$ depends on the presence of many small, highly-connected components in this network. Concerning the comparison with Lemon (Table 11), it is evident and even more significant the lack of similarity between ML-LCD and the competing one: mean similarities are occasionally above 0.2 , while percentages of identical communities are zero in nearly all cases, again with the exception of $D B L P$ (in which case, they are around or below $5 \%$ ).

Overall, not surprisingly, the above results confirm that accounting for the multiple available layer information, as our ML-LCD methods do, brings to the identification of local communities that are quite different from the ones obtained by a single-layer LCD method applied on the aggregate graph derived from the original multilayer network.

\subsection{Comparison with global community detection methods}

Table 12 summarizes statistics on the number of communities identified by LocToGlob (with suffixes $l w$, wlsim, and clsim to distinguish among the different variants) and by the competing methods - please note that we do not report results of PMM since, for this method, the number of communities is an input parameter. We observe that, among our proposed methods, LocToGlob$l w$ and LocToGlob-wlsim tend to produce the lowest and highest number of communities, respectively (which is in line with results previously presented). Considering also LART and GL, the former yields a number of communities 
Table 13 Multilayer modularity performance of global community detection methods. Bold values and underlined values correspond to the highest per-dataset modularities considering ML-LCD methods and all methods, respectively.

\begin{tabular}{|c|c|c|c|c|c|c|c|c|}
\hline \multirow{2}{*}{\multicolumn{2}{|c|}{ Dataset }} & \multicolumn{2}{|c|}{ LocToGlob- $l w$} & \multicolumn{2}{|c|}{ LocToGlob-wlsim } & \multicolumn{3}{|c|}{ LocToGlob-clsim } \\
\hline & & mean & $\max$ & mean & $\max$ & mean & \multicolumn{2}{|c|}{$\max$} \\
\hline \multicolumn{2}{|l|}{ Airlines } & 0.033 & 0.040 & 0.023 & 0.028 & 0.027 & \multicolumn{2}{|c|}{0.033} \\
\hline \multicolumn{2}{|l|}{$A U C S$} & $\begin{array}{l}0.225 \\
0.113\end{array}$ & 0.252 & 0.212 & 0.233 & 0.214 & \multicolumn{2}{|c|}{0.239} \\
\hline \multicolumn{2}{|l|}{ Biogrid } & & 0.135 & 0.074 & 0.076 & 0.085 & \multicolumn{2}{|c|}{0.088} \\
\hline RemoteSensin & & 0.196 & \multirow{2}{*}{$\begin{array}{l}0.201 \\
0.281\end{array}$} & 0.185 & 0.190 & 0.151 & \multicolumn{2}{|c|}{0.157} \\
\hline \multicolumn{2}{|c|}{ RealityMining } & 0.243 & & \multirow{2}{*}{$\begin{array}{l}0.209 \\
0.118\end{array}$} & 0.226 & \multirow{2}{*}{$\begin{array}{l}0.1017 \\
0.117\end{array}$} & \multirow{2}{*}{\multicolumn{2}{|c|}{$\begin{array}{l}0.225 \\
0.122 \\
\end{array}$}} \\
\hline$T W-Y T-F F$ & & $\underline{0.167}$ & 0.185 & & 0.121 & & & \\
\hline Dataset & LART & GL & & MM-1 & PMI & & & $\mathrm{n}-3$ \\
\hline & & & me & $\max$ & mean & $\max$ & mean & $\max$ \\
\hline Airlines & 0.013 & $\underline{0.037}$ & 0.0 & 0.018 & 0.014 & 0.016 & 0.014 & 0.015 \\
\hline$A U C S$ & 0.154 & $\underline{0.249}$ & 0.1 & 0.222 & 0.157 & 0.187 & 0.166 & 0.218 \\
\hline Biogrid & NA & $\underline{0.225}$ & 0.0 & 0.039 & 0.037 & 0.039 & 0.038 & 0.039 \\
\hline RemoteSensing & 0.069 & $\underline{0.269}$ & 0.1 & 0.167 & 0.152 & 0.161 & 0.143 & 0.150 \\
\hline RealityMining & 0.101 & $\underline{0.288}$ & 0.1 & 0.217 & 0.165 & 0.200 & 0.171 & 0.237 \\
\hline$T W-Y T-F F$ & NA & 0.022 & 0.0 & 0.580 & 0.060 & 0.069 & 0.053 & 0.053 \\
\hline
\end{tabular}

that is significantly higher than that of other methods; however, on Biogrid and $T W-Y T-F F$, it was not able to terminated as it incurred an out-of-memory issue 4 Note also that on Biogrid, all the methods produced the largest number of communities, which might be explained since this network is characterized by the highest sparsity among all the employed datasets (cf. Table 1).

Table 13 reports multilayer modularity values corresponding to the global, non-overlapping community structures obtained by LocToGlob and competing methods. Notation PMM- $i$, with $i=\{1,2,3\}$, refers to the setting of number of communities corresponding to each of the LocToGlob methods (cf. Sect. 4.3) ${ }^{5}$

A first important remark is that, on all network datasets, LocToGlob$l w$ always leads to better modularities than the other two approaches, with LocToGlob-wlsim and LocToGlob-clsim showing similar behaviors. Compared to the competitors, all LocToGlob methods outperform, in terms of both average and maximum modularity, LART and the three variants of PMM. In the case of GL, LocToGlob-lw obtains comparable average modularity; moreover, LocToGlob-lw achieves higher maximum modularity on AUCS, Airlines and $T W-Y T-F F$ with respect to $\mathrm{GL}$.

The obtained results highlight that our proposed framework is able to reach comparable or even better modularity results against state-of-the-art global community detection methods. We stress here that this evaluation setting is actually a severe comparison for our approach since: all the competitors exploit a complete knowledge of the graph to optimize their internal objective function and, the multilayer modularity we employ as evaluation criterion is also the function internally optimized by GL.

\footnotetext{
${ }^{4}$ We do not include $D B L P$ in this evaluation because the competitors incurred out-ofmemory issues, though our methods did not.

5 For LART and GL we report only one performance score since they have a deterministic behavior.
} 


\section{Conclusion}

We addressed the novel problem of local community detection in multilayer networks, and presented the first algorithmic framework to solve it. Our MLLCD formulation relies on the optimization of a function based on internal and external connectivity of a multilayer local community, and is instantiated into three methods that differ in the way within- and across-layer features are taken into account to discover a local community for a given seed node. An extensive analysis over seven real-world multilayer networks has shown significance and ability of our methods in detecting multilayer local communities. In addition, by inferring a global non-overlapping community structure from the local communities identified for a given set of seed nodes in the network, we demonstrated that our ML-LCD methods can produce higher-modularity communities than state-of-the-art methods designed for multilayer global community detection.

Our future research directions would move along paths involving both theoretical and experimental aspects. On the former, we would like to study alternative objective functions for the ML-LCD problem and theoretical guarantees on the sub-optimality of the solutions. Also, an interesting aspect to understand is how outlier and hub nodes can be efficiently detected and handled during the construction of a local community. Considering the experimental evaluation, it would be an important addiction the construction of a groundtruth for the local communities to be identified in a multilayer network. Finally, we envisage a number of application problems for which ML-LCD methods can profitably be used, such as friendship prediction, targeted influence propagation, and more in general, mining in incomplete networks.

Acknowledgements D. Ienco would like to acknowledge the National French Center for Spatial Study (CNES), in the framework of the project "DYNAMITEF TOSCA 2016", to support this study with the RemoteSensing dataset.

\section{References}

M. Berlingerio, M. Coscia, F. Giannotti, Finding and Characterizing Communities in Multidimensional Networks, in Proc. IEEE/ACM Int. Conf. on Advances in Social Networks Analysis and Mining (ASONAM), 2011, pp. 490-494

M. Berlingerio, F. Pinelli, F. Calabrese, ABACUS: frequent pattern mining-based community discovery in multidimensional networks. Data Min. Knowl. Discov. 27(3), 294-320 (2013)

B. Boden, S. Günnemann, H. Hoffmann, T. Seidl, Mining Coherent Subgraphs in Multi-layer Graphs with Edge Labels, in Proc. ACM SIGKDD Int. Conf. on Knowledge Discovery and Data Mining (KDD), 2012, pp. 1258-1266

F. Bonchi, A. Gionis, F. Gullo, A. Ukkonen, Distance oracles in edge-labeled graphs, in Proc. Int. Conf. on Extending Database Technology (EDBT), 2014, pp. 547-558

R. Bourqui, D. Ienco, A. Sallaberry, P. Poncelet, Multilayer Graph Edge Bundling, in Proc. IEEE Pacific Visualization Symposium (PacificVis), 2016, pp. 184-188

K. Branting, Context-sensitive detection of local community structure. Social Netw. Analys. Mining 2(3), 279-289 (2012) 
D. Cai, Z. Shao, X. He, X. Yan, J. Han, Community Mining from Multi-relational Networks, in Proc. European Conf. on Principles and Practice of Knowledge Discovery in Databases (PKDD), 2005, pp. 445-452

V. Carchiolo, A. Longheu, M. Malgeri, G. Mangioni, Communities Unfolding in Multislice Networks, in Proc. Complex Networks, 2010, pp. 187-195

A. Cardillo, J. Gomez-Gardenes, M. Zanin, M. Romance, D. Papo, F. del Pozo, S. Boccaletti, Emergence of network features from multiplexity. Scientific Reports 3:1344 (2013)

J. Chen, O.R. Zaïane, R. Goebel, Local Community Identification in Social Networks, in Proc. IEEE/ACM Int. Conf. on Advances in Social Networks Analysis and Mining (ASONAM), 2009, pp. 237-242

A. Clauset, Finding local community structure in networks. Phys. Rev. E 72(2), 026132 (2005)

M.E. Dickison, M. Magnani, L. Rossi, Multilayer Social Networks (Cambridge University Press, UK, 2016)

J. Fagnan, O.R. Zaïane, D. Barbosa, Using triads to identify local community structure in social networks, in Proc. IEEE/ACM Int. Conf. on Advances in Social Networks Analysis and Mining (ASONAM), 2014, pp. 108-112

M. Hmimida, R. Kanawati, Community detection in multiplex networks: A seed-centric approach. Networks and Heterogeneous Media 10(1), 71-85 (2015)

L.G.S. Jeub, M.W. Mahoney, P.J. Mucha, M.A. Porter, A Local Perspective on Community Structure in Multilayer Networks. Network Science, 1-20 (2015)

R. Kanawati, Empirical evaluation of applying ensemble methods to ego-centred community identification in complex networks. Neurocomputing 150, 417-427 (2015)

J. Kim, J.- Lee, Community detection in multi-layer graphs: A survey. SIGMOD Record 44(3), 37-48 (2015)

M. Kivela, A. Arenas, M. Barthelemy, J.P. Gleeson, Y. Moreno, M.A. Porter, Mutilayer networks. Journal of Complex Networks 2(3), 203-271 (2014)

Z. Kuncheva, G. Montana, Community Detection in Multiplex Networks using Locally Adaptive Random Walks, in Proc. IEEE/ACM Int. Conf. on Advances in Social Networks Analysis and Mining (ASONAM), 2015, pp. 1308-1315

Y. Li, K. He, D. Bindel, J.E. Hopcroft, Uncovering the Small Community Structure in Large Networks: A Local Spectral Approach, in Proc. ACM Conf. on World Wide Web $(W W W), 2015$, pp. 658-668

C.W. Loe, H.J. Jensen, Comparison of communities detection algorithms for multiplex. Physica A 431, 29-45 (2015)

T. Michoel, B. Nachtergaele, Alignment and integration of complex networks by hypergraphbased spectral clustering. Phis. Rev. E 85:056111 (2012)

P.J. Mucha, T. Richardson, K. Macon, M.A. Porter, J.-P. Onnela, Community structure in time-dependent, multiscale, and multiplex networks. Science 328(5980), 876-878 (2010)

M.E.J. Newman, M. Girvan, Finding and evaluating community structure in networks. Phys. Rev. E 69(2), 026113 (2004)

E.E. Papalexakis, L. Akoglu, D. Ienco, Do more views of a graph help? Community detection and clustering in multi-graphs, in Proc. Int. Conf. on Information Fusion, 2013, pp. 899-905

L. Tang, X. Wang, H. Liu, Uncovering Groups via Heterogeneous Interaction Analysis, in Proc. ICDM, 2009, pp. 503-512

L. Tang, X. Wang, H. Liu, Community detection via heterogeneous interaction analysis. Data Min. Knowl. Discov. 25, 1-33 (2012)

Z. Yakoubi, R. Kanawati, LICOD: A leader-driven algorithm for community detection in complex networks. Vietnam J. Computer Science 1(4), 241-256 (2014)

A. Zakrzewska, D.A. Bader, A Dynamic Algorithm for Local Community Detection in Graphs, in Proc. IEEE/ACM Int. Conf. on Advances in Social Networks Analysis and Mining (ASONAM), 2015, pp. 559-564 